\title{
A POSTERIORI ERROR ANALYSIS OF FINITE ELEMENT METHOD FOR LINEAR NONLOCAL DIFFUSION AND PERIDYNAMIC MODELS
}

\author{
QIANG DU, LILI JU, LI TIAN, AND KUN ZHOU
}

\begin{abstract}
In this paper, we present some results on a posteriori error analysis of finite element methods for solving linear nonlocal diffusion and bond-based peridynamic models. In particular, we aim to propose a general abstract frame work for a posteriori error analysis of the peridynamic problems. A posteriori error estimators are consequently prompted, the reliability and efficiency of the estimators are proved. Connections between nonlocal a posteriori error estimation and classical local estimation are studied within continuous finite element space. Numerical experiments (1D) are also given to test the theoretical conclusions.
\end{abstract}

\section{INTRODUCTION}

Since peridynamic (PD) models were first introduced by Silling in [34, their effectiveness has been demonstrated in a number of applications, e.g., fracture and failure of composites, fracture of polycrystals, nanofiber networks and so on, where numerical simulations of singular behavior such as crack growth or damage are involved. Peridynamics is becoming an attractive emerging tool for the multiscale material simulations. On one hand, it is an integral-type nonlocal continuum theory which provides a more general set-up than the classical partial differential equation (PDE) based elasticity theories for physical problems with discontinuities. On the other hand, it can also be viewed as a continuum version of molecular dynamics.

Along with research efforts on the modeling and application to practical problems, there have been some recent studies concerning the mathematical and numerical analysis of PD models. For example, some well-posedness studies of Cauchy problems for linear PD models have been given in [4, 24, 25, 26, 27, 28. Similar studies for initial and nonlocal boundary value problems have been considered in [40] which has also investigated the issue of convergence of finite dimensional approximations of the linear bond-based PD models. There have been a variety of numerical methods implemented for the approximations of PD models including finite difference, finite element, quadrature and particle based methods [2, 6, 14, 20, 32, 36]. Convergence analysis and conditioning estimates for the discretized system have been given in [2, 3, 23, 40. Moreover, connections between linear PD models and

Received by the editor April 27, 2011 and, in revised form, March 6, 2012.

2010 Mathematics Subject Classification. 65J15, 65R20, 65N30, 65 N15.

Key words and phrases. Peridynamic models, nonlocal diffusion, a posteriori error estimate, finite element.

This work was supported in part by the U.S. Department of Energy Office of Science under grant number DE-SC0005346 and by the U.S. National Science Foundation under grant number DMS-1016073. 
the continuum elasticity models have been established in [25, 26, 37, 40] as the ratio of the PD horizon to the effective length scale decreases. In [29, a nonlocal vector calculus was developed for the study of boundary value problems of scalar nonlocal diffusion equations. Extensions to vector and tensor fields and applications to nonlocal systems of variational equations and abstract nonlocal balance laws including the peridynamic models are presented in 22 . The framework presented in 22 has been utilized in recent mathematical and numerical analysis of nonlocal variational problems [23, 24]. Our current study is a part of the ongoing efforts.

In this work, we continue the works started in [40] and [23] on the numerical analysis of finite element method for bond-based linear steady state PD models and the associated nonlocal diffusion equations. Motivated by the need of adaptive computation for large scale simulations based on the nonlocal models with possible singular solutions, we mainly focus, in this work, on the development of a posteriori error estimators for nonlocal model equations. And we aim to provide more theoretical guidance on the design of automatic error control and adaptive numerical schemes. Historically, a posteriori error estimations have played a very important role in adaptive mesh generation and algorithm design for numerical PDEs. Theoretical and systematical study of a posteriori error estimators for finite element approximation began in the late 1970's [11, and since then there have been a lot of studies in the literature; see for example, 1, 8, 9, 10, 12, 18, 21, 30, 38, and the references cited therein. There have also been many works on adaptive methods and a posteriori analysis for integral equations; see [17, 19, 33. While adaptive refinement and coarsening methods for PD models have been studied in [13, 14, 35, 39, there has not been any serious attempt to derive rigorous a posteriori error estimators. On the other hand, although PD models are integral equations, given the variational formulations presented in 22] based on the framework of nonlocal calculus and nonlocal balance laws, there is a close resemblance between the nonlocal PD models and the classical differential equations, which can be utilized to develop a posteriori error estimates for PD models. The latter is the main objectives of the current work. We provide a rigorous a posteriori error estimator which is shown to be both reliable and efficient. Moreover, we pay close attention to not only the connections but also the key differences between the a posterior analysis of local and nonlocal models. Dependence of the error estimators on the model parameters is also considered. Some preliminary numerical experiments provide further substantiation of our theoretical analysis.

The paper is organized as follows. To prepare for the necessary background, we present in Section 2 some definitions of the nonlocal operators as constructed in 22] and describe the basic model equations. We also briefly recall the key features of the a posteriori error analysis for PDE models so as to offer contrast to the framework developed here for the nonlocal models. In order to present the essential features of the analysis without too many technical complications, we begin our analysis in a simpler setting, namely, we first consider a linear scalarvalued nonlocal diffusion model in Section 3. An a posteriori error estimator for such a model is introduced and its local limit is analyzed. Then in Section 4, we move on to develop a posteriori estimators for bond-based linear PD models in an analogous manner but with a focus on treating the extra complication due to the system nature. We present several numerical experiments in Section 5 to test our theories, and some conclusions are given in Section 6 . 


\section{Nonlocal operators AND MOdel EQUATIONS}

Let us first recall some nonlocal operators as introduced in 22 . Let $\Omega$ be a bounded, open, connected spatial domain in $\mathbb{R}^{d}$ and

$$
\Omega=\left(\Omega_{s} \cup \Omega_{c}\right) \cup\left(\bar{\Omega}_{s} \cap \bar{\Omega}_{c}\right)^{o},
$$

where $\Omega_{s}$ denotes the solution domain, $\Omega_{c}$ denotes the constraint domain such that $\Omega_{s} \cap \Omega_{c}=\emptyset$ and $\left(\bar{\Omega}_{s} \cap \bar{\Omega}_{c}\right)^{o}$ denotes the interior of the region $\bar{\Omega}_{s} \cap \bar{\Omega}_{c}$. We assume that $\Omega_{s}$ has convex polygonal boundaries with $\Omega_{c}$ being a domain surrounding $\Omega_{s}$.

Let $\beta\left(\mathbf{x}, \mathbf{x}^{\prime}\right): \Omega \times \Omega \rightarrow \mathbb{R}$ be a scalar nonnegative two-point function which is symmetric, that is, $\beta\left(\mathbf{x}, \mathbf{x}^{\prime}\right)=\beta\left(\mathbf{x}^{\prime}, \mathbf{x}\right)$, and has a compact support such that

$$
\beta\left(\mathbf{x}, \mathbf{x}^{\prime}\right)=0 \quad \forall \mathbf{x}, \mathbf{x}^{\prime} \in \Omega, \text { with }\left|\mathbf{x}^{\prime}-\mathbf{x}\right|>\delta
$$

for some constant number $\delta>0$. We also assume it is nondegenerate in the sense that there exists a constant $\beta_{0}(\delta)>0$ such that

$$
\beta\left(\mathbf{x}, \mathbf{x}^{\prime}\right) \geq \beta_{0}(\delta) \quad \forall \mathbf{x}, \mathbf{x}^{\prime} \in \Omega, \text { with }\left|\mathbf{x}^{\prime}-\mathbf{x}\right|<\delta / 2 .
$$

Here, $\delta / 2$ may be replaced by other suitable constants smaller than $\delta$ to ensure the nondegeneracy of $\beta\left(\mathbf{x}, \mathbf{x}^{\prime}\right)$. For convenience of reference, we call $\delta$ the horizon parameter, following the naming convention given in 34 .

Let $\boldsymbol{\alpha}\left(\mathbf{x}, \mathbf{x}^{\prime}\right): \Omega \times \Omega \rightarrow \mathbb{R}^{d}$ be a skew-symmetric two-point mapping such that

$$
\boldsymbol{\alpha}\left(\mathbf{x}^{\prime}, \mathbf{x}\right)+\boldsymbol{\alpha}\left(\mathbf{x}, \mathbf{x}^{\prime}\right)=0 .
$$

Given a two-point function $\mathbf{v}: \Omega \times \Omega \rightarrow \mathbb{R}^{d}$ and a one-point function $u: \Omega \rightarrow \mathbb{R}$, the nonlocal point divergence operator $\mathcal{D}(\mathbf{v}): \Omega \rightarrow \mathbb{R}$ is defined as (see [22])

$$
\mathcal{D}(\mathbf{v})(\mathbf{x}):=-\int_{\Omega}\left(\mathbf{v}\left(\mathbf{x}, \mathbf{x}^{\prime}\right)+\mathbf{v}\left(\mathbf{x}^{\prime}, \mathbf{x}\right)\right) \cdot \boldsymbol{\alpha}\left(\mathbf{x}, \mathbf{x}^{\prime}\right) d \mathbf{x}^{\prime}, \quad \forall \mathbf{x} \in \Omega
$$

and the adjoint operator $\mathcal{D}^{*}(u): \Omega \times \Omega \rightarrow \mathbb{R}^{d}$ of $\mathcal{D}$ is given by

$$
\mathcal{D}^{*}(u)\left(\mathbf{x}, \mathbf{x}^{\prime}\right):=\left(u\left(\mathbf{x}^{\prime}\right)-u(\mathbf{x})\right) \boldsymbol{\alpha}\left(\mathbf{x}, \mathbf{x}^{\prime}\right), \quad \forall \mathbf{x}^{\prime}, \mathbf{x} \in \Omega .
$$

2.1. Nonlocal diffusion equations. In Section [3, we consider the nonlocal constrained-value problem

$$
\left\{\begin{aligned}
\mathcal{D}\left(\beta \mathcal{D}^{*}(u(\mathbf{x}))\right) & =f(\mathbf{x}) & & \text { for } \mathbf{x} \in \Omega_{s}, \\
u(\mathbf{x}) & =0 & & \text { for } \mathbf{x} \in \Omega_{c},
\end{aligned}\right.
$$

where the first equation is called a nonlocal diffusion equation, while the second equation is a nonlocal homogeneous Dirichlet constraint (nonlocal boundary condition). The above problem has a natural variational characterization [23, 24] and the natural function space associated with the solution of (2.4) is given by

$$
V_{c}:=\left\{u \in L^{2}(\Omega): \int_{\Omega} \int_{\Omega} \beta\left(\mathbf{x}, \mathbf{x}^{\prime}\right)\left|\mathcal{D}^{*}(u)\left(\mathbf{x}, \mathbf{x}^{\prime}\right)\right|^{2} d \mathbf{x}^{\prime} d \mathbf{x}<\infty, u(\mathbf{x})=0 \forall \mathbf{x} \in \Omega_{c}\right\} .
$$

More precise characterizations of $V_{c}$ are given in later sections. 
2.2. Nonlocal bond-based linear peridynamic models. While the scalar nonlocal diffusion equations have many applications on their own [16, the vectorized form of (2.4) can have the same form as that of a linear bond-based peridynamic model. The latter may be more conveniently expressed with some additional operators in the nonlocal vector calculus framework. Indeed, given the scalar two point mapping $v: \Omega \times \Omega \rightarrow \mathbb{R}$, the nonlocal point gradient operator $\mathcal{G}(v): \Omega \rightarrow \mathbb{R}^{d}$ is defined as

$$
\mathcal{G}(v)(\mathbf{x}):=-\int_{\Omega}\left(v\left(\mathbf{x}, \mathbf{x}^{\prime}\right)+v\left(\mathbf{x}^{\prime}, \mathbf{x}\right)\right) \boldsymbol{\alpha}\left(\mathbf{x}, \mathbf{x}^{\prime}\right) d \mathbf{x}^{\prime}, \quad \forall \mathbf{x} \in \Omega .
$$

Given the point mapping $\mathbf{u}(\mathbf{x}): \Omega \rightarrow \mathbb{R}^{d}$, the adjoint operator $\mathcal{G}^{*}(\mathbf{u}): \Omega \times \Omega \rightarrow \mathbb{R}$ corresponding to the point operator $\mathcal{G}$ defined above is given by

$$
\mathcal{G}^{*}(\mathbf{u})\left(\mathbf{x}, \mathbf{x}^{\prime}\right):=\left(\mathbf{u}\left(\mathbf{x}^{\prime}\right)-\mathbf{u}(\mathbf{x})\right) \cdot \boldsymbol{\alpha}\left(\mathbf{x}, \mathbf{x}^{\prime}\right), \quad \forall \mathbf{x}, \mathbf{x}^{\prime} \in \Omega .
$$

Following the discussions in 22, 24, given the $\beta\left(\mathbf{x}, \mathbf{x}^{\prime}\right)$ and $\boldsymbol{\alpha}$ defined above, we may have the following nonlocal constrained-value problem for the bond-based linear peridynamic model

$$
\left\{\begin{aligned}
\mathcal{G}\left(\beta \mathcal{G}^{*}(\mathbf{u}(\mathbf{x}))\right) & =\mathbf{f}(\mathbf{x}) \quad \text { for } \mathbf{x} \in \Omega_{s} \\
\mathbf{u}(\mathbf{x}) & =0 \quad \text { for } \mathbf{x} \in \Omega_{c} .
\end{aligned}\right.
$$

2.3. More discussions on the nonlocal model problems. For both the model equations (2.4) and (2.8), it is straightforward to write down their variational form using the nonlocal operators and the nonlocal balance laws [23, 24]. This in turn allows the construction of Galerkin approximations using, for instance, finite element spaces.

For these model equations, the properties of solutions of the model equations depend crucially on the kernel function $\gamma=\boldsymbol{\alpha} \cdot \boldsymbol{\alpha} \beta$. In the literature, a type of kernel functions that have been most-widely studied is the class of nonnegative integrable kernels.

Here, we make a simplifying assumption that the kernel function $\gamma: \Omega \times \Omega \rightarrow \mathbb{R}$ is a function which only depends on $\mathbf{z}=\mathbf{x}^{\prime}-\mathbf{x}$. Moreover, the integrable kernel function is assumed to satisfy the property that there exist positive constants $c(\delta)$ and $M(\delta)$ such that

$$
\begin{array}{r}
0<c(\delta) \leq \int_{B_{\delta}(0)} \gamma(\mathbf{z}) d \mathbf{z} \leq M(\delta)<\infty, \\
\gamma(\mathbf{z}) \geq 0 \quad \forall|\mathbf{z}| \leq \delta, \quad \gamma(\mathbf{z})=0 \quad \forall|\mathbf{z}|>\delta .
\end{array}
$$

More general and nonintegrable kernels can also be considered [25]. The nonintegrable case corresponds to a broader class of problems which, for instance in the peridynamic context, still retains well-defined elastic moduli and leads to smoothing effect of the solution operators. However, the case corresponding to (2.9) is of particular interest in many practical applications of the peridynamic modeling of materials. Moreover, for $f \in L^{2}(\Omega)$, given the assumption (2.9), one expects that the solutions of (2.4) and (2.8) are in $L^{2}(\Omega)$ and $\left(L^{2}(\Omega)\right)^{d}$, respectively, so that the solution operators do not have any extra smoothing effect. This situation sets such nonlocal models apart from their local limit and is thus the main focus of the present study. We refer to [3, 5, 23, 29, 40, for additional discussions of the well-posedness for the scalar case of the model equations and [24, 40] on PD statebased and bond-based systems. To avoid some technical complications which are 
not important for practical applications, additional assumptions are made in later sections to simplify the analysis.

2.4. Finite element discretization and a posteriori error analysis of the classic diffusion problem. We now briefly recall a posteriori error estimate for PDE problems. We choose to focus on the residual-based a posteriori error estimate which has been discussed extensively in the literature; see, e.g., 38. We take, for example, the following model equation:

$$
\left\{\begin{aligned}
-\nabla \cdot(\mathbb{D} \nabla u(x)) & =f(x) \quad \text { for } x \in \Omega_{s}, \\
u(x) & =0 \quad \text { for } x \in \partial \Omega_{s},
\end{aligned}\right.
$$

where we assume that $f \in L^{2}\left(\Omega_{s}\right)$ and $\mathbb{D}(\mathbf{x})$ is a $d \times d$ symmetric positive definite matrix for any $\mathbf{x} \in \Omega_{s}$. We use $u$ to denote the exact solution of the PDE problem (2.10).

Now, let $\{K\}$ denote a spatial simplicial mesh of $\Omega_{s}\left(\Omega_{s}=\bigcup K\right)$ and let $\mathbf{S}_{\text {local }}^{h}$ denote a finite dimensional subspace of $H_{0}^{1}\left(\Omega_{s}\right)$ defined with respect to the mesh $\{K\}$. That is, $\mathbf{S}_{\text {local }}^{h}$ denotes a space of continuous and piecewise smooth functions (polynomials) defined on $\{K\}$, and $u^{h}$ is the Galerkin finite dimensional approximation of $u$ in the subspace $\mathbf{S}_{\text {local }}^{h}$ defined by the following problem:

$$
\left\{\begin{array}{l}
\text { Find } u^{h} \in \mathbf{S}_{\text {local }}^{h}, \text { such that for any } v^{h} \in \mathbf{S}_{\text {local }}^{h}, \\
\int_{\Omega_{s}} \mathbb{D} \nabla u^{h} \cdot \nabla v^{h} d \mathbf{x}=\int_{\Omega_{s}} f v^{h} d \mathbf{x}, \\
\text { and } u^{h}(\mathbf{x})=0 \text { on } \partial \Omega_{s} .
\end{array}\right.
$$

To obtain a posteriori error estimate for (2.11), we apply integration by parts on each mesh element $K$ which gives that for any $v \in H_{0}^{1}\left(\Omega_{s}\right)$,

$$
\begin{array}{rl}
\int_{\Omega_{s}} & \mathbb{D} \nabla\left(u-u^{h}\right) \cdot \nabla v d \mathbf{x} \\
\quad= & \sum_{K}\left(\int_{K}\left(f+\nabla \cdot\left(\mathbb{D} \nabla u^{h}\right)\right) v d \mathbf{x}-\frac{1}{2} \int_{\partial K} \llbracket \mathbb{D} \nabla u^{h} \rrbracket_{\mathbf{r}} v d \mathbf{s}\right),
\end{array}
$$

where $\sigma$ is the set of all the interior edges (faces) of the mesh; $\llbracket \mathbb{D} \nabla u^{h} \rrbracket_{\mathbf{r}}$ denotes the normal jump of $\mathbb{D} \nabla u^{h}$ on an edge (face) $\mathbf{r}$ as defined by

$$
\llbracket \mathbb{D} \nabla u^{h} \rrbracket_{\mathbf{r}}=\left.\mathbb{D} \nabla u_{K}^{h}\right|_{\mathbf{r}} \cdot \overrightarrow{\mathbf{n}}_{K}+\left.\mathbb{D} \nabla u_{K^{\prime}}^{h}\right|_{\mathbf{r}} \cdot \overrightarrow{\mathbf{n}}_{K^{\prime}},
$$

where $\mathbf{r}$ is the common edge (face) of $K$ and $K^{\prime}$ with outward normal $\overrightarrow{\mathbf{n}}_{K}$ and $\overrightarrow{\mathbf{n}}_{K^{\prime}}$, respectively; $u_{K}^{h}$ and $u_{K^{\prime}}^{h}$ denote $\left.u^{h}\right|_{K}$ and $\left.u^{h}\right|_{K^{\prime}}$, respectively.

In the classical residual-based a posteriori error analysis for (2.11), by making use of (2.12), the interior residual is defined by

$$
r_{*}^{h}=f+\nabla \cdot\left(\mathbb{D} \nabla u^{h}\right) \text { in } K,
$$

and the boundary residual by

$$
R_{*}^{h}=\llbracket \mathbb{D} \nabla u^{h} \rrbracket_{\mathbf{r}} \quad \text { on any interior edge(face) } \mathbf{r} .
$$

Then, the local a posteriori error estimator (energy type) $\eta_{K}$ associated with element $K$ can be defined as

$$
\eta_{K}^{2}=h_{K}^{2}\left\|r_{*}^{h}\right\|_{L^{2}(K)}^{2}+h_{K}\left\|R_{*}^{h}\right\|_{L^{2}(\partial K)}^{2},
$$


where $h_{K}$ denotes the size (mesh parameter) of $K$. The reliability and efficiency of the estimator have been proved in [11, i.e., the estimator neither underestimates nor overestimates the actual error $u-u^{h}$.

In the next section, we mimic the above framework to get a posteriori error approximation for some nonlocal linear equations. We note that our study also shows significant differences between a posteriori error analysis for the nonlocal and local models. Since applying a nonlocal operator to a piecewise polynomial, which is not globally smooth, could lead to a very complicated result, therefore instead of using approximation of interpolations, we use the equivalence of energy space and its dual space in the analysis. Then by using Taylor expansion, changing the order of integrations and studying the flux on interior edges, we prove the weak convergence of the nonlocal error estimator to its local counterpart, which is the a posteriori error estimator for the corresponding $\mathrm{PDE}$, as $\delta \rightarrow 0$.

\section{Finite element discretization And a posteriori ERror analysis of LINEAR NONLOCAL DIFFUSION EQUATIONS}

We now study the finite element discretization of (2.4).

3.1. A nonlocal bilinear form and the weak formulation. We first prompt the following lemmas.

Lemma 3.1. Assume the kernel function $\gamma$ satisfies (2.9), then for any $u \in L^{2}(\Omega)$, we have

$$
\mathcal{D}\left(\beta \mathcal{D}^{*}(u)\right) \in L^{2}(\Omega)
$$

Proof. For $u \in L^{2}(\Omega)$, we have, by applying Cauchy-Schwarz inequality,

$$
\begin{aligned}
\| \mathcal{D} & \left(\beta \mathcal{D}^{*}(u)\right) \|_{L^{2}(\Omega)}^{2} \\
& =\int_{\Omega}\left|\int_{\Omega} 2 \gamma\left(u\left(\mathbf{x}^{\prime}\right)-u(\mathbf{x})\right) d \mathbf{x}^{\prime}\right|^{2} d \mathbf{x} \\
& \leq 4 \int_{\Omega} \int_{\Omega} \gamma d \mathbf{x}^{\prime} \int_{\Omega} \gamma\left(u\left(\mathbf{x}^{\prime}\right)-u(\mathbf{x})\right)^{2} d \mathbf{x}^{\prime} d \mathbf{x} \\
& =4 \int_{\Omega} \gamma(\mathbf{z}) d z \int_{\Omega} \int_{\Omega} \gamma\left(u\left(\mathbf{x}^{\prime}\right)-u(\mathbf{x})\right)^{2} d \mathbf{x}^{\prime} d \mathbf{x} \\
& =4 \int_{B_{\delta}(0)} \gamma(\mathbf{z}) d \mathbf{z} \int_{\Omega} \int_{\Omega} \gamma\left(u^{2}\left(\mathbf{x}^{\prime}\right)+u^{2}(\mathbf{x})-2 u\left(\mathbf{x}^{\prime}\right) u(\mathbf{x})\right) d \mathbf{x}^{\prime} d \mathbf{x} \\
& =8 \int_{B_{\delta}(0)} \gamma(\mathbf{z}) d \mathbf{z}\left(\int_{\Omega} \int_{\Omega} \gamma u^{2}(\mathbf{x}) d \mathbf{x}^{\prime} d \mathbf{x}-\int_{\Omega} \int_{\Omega} \gamma u\left(\mathbf{x}^{\prime}\right) u(\mathbf{x}) d \mathbf{x}^{\prime} d \mathbf{x}\right),
\end{aligned}
$$

where

$$
\int_{\Omega} \int_{\Omega} \gamma u^{2}(\mathbf{x}) d \mathbf{x}^{\prime} d \mathbf{x} \leq \int_{B_{\delta}(0)} \gamma(\mathbf{z}) d \mathbf{z} \cdot\|u\|_{L^{2}(\Omega)}^{2}
$$

and 


$$
\begin{aligned}
& \int_{\Omega} \int_{\Omega} \gamma u\left(\mathbf{x}^{\prime}\right) u(\mathbf{x}) d \mathbf{x}^{\prime} d \mathbf{x} \\
& \quad \leq\left(\int_{\Omega} \int_{\Omega} \gamma u^{2}\left(\mathbf{x}^{\prime}\right) d \mathbf{x}^{\prime} d \mathbf{x}\right)^{1 / 2}\left(\int_{\Omega} \int_{\Omega} \gamma u^{2}(\mathbf{x}) d \mathbf{x}^{\prime} d \mathbf{x}\right)^{1 / 2} \\
& \quad \leq \int_{B_{\delta}(0)} \gamma(\mathbf{z}) d \mathbf{z} \cdot\|u\|_{L^{2}(\Omega)}^{2},
\end{aligned}
$$

then the conclusion follows.

Lemma 3.2. Assume the kernel function $\gamma$ satisfies (2.9), then for any $u, v \in$ $L_{n 0}^{2}(\Omega)$,

$$
\int_{\Omega_{s}} v \mathcal{D}\left(\beta \mathcal{D}^{*}(u)\right) d \mathbf{x}-\int_{\Omega} \int_{\Omega} \mathcal{D}^{*}(v) \beta \mathcal{D}^{*}(u) d \mathbf{x}^{\prime} d \mathbf{x}=0 .
$$

Proof. For any $u, v \in L_{n 0}^{2}(\Omega)$, considering Lemma 3.1 and nonlocal integration by parts as shown in 22, we then have

$$
\int_{\Omega} \int_{\Omega} \mathcal{D}^{*}(v) \beta \mathcal{D}^{*}(u) d \mathbf{x}^{\prime} d \mathbf{x}=\int_{\Omega} v \mathcal{D}\left(\beta \mathcal{D}^{*}(u)\right) d \mathbf{x},
$$

so (3.5) follows by considering constraint of $v$ on $\Omega_{c}$.

We may define a nonlocal bilinear form

$$
B(u, v)=\int_{\Omega} \int_{\Omega} \mathcal{D}^{*}(u) \beta \mathcal{D}^{*}(v) d \mathbf{x}^{\prime} d \mathbf{x},
$$

for any $u, v \in V_{c}$.

Under the assumption (2.9), $\mathcal{D}\left(\beta \mathcal{D}^{*}(\cdot)\right)$ defines a nonlocal diffusion operator from $L^{2}(\Omega)$ to $L^{2}(\Omega)$, and one expects to get the boundedness and coercivity of $B(\cdot, \cdot)$ in the space

$$
V_{c}=L_{n 0}^{2}(\Omega):=\left\{v \in L^{2}\left(\Omega_{s}\right): v(\mathbf{x})=0 \text { for } \mathbf{x} \in \Omega_{c}\right\},
$$

that is, for all $u, v \in L_{n 0}^{2}(\Omega)$, there exists a positive constant $C_{1}(\delta)$ such that

$$
|B(u, v)| \leq 4 M(\delta)\|u\|_{L^{2}(\Omega)}\|v\|_{L^{2}(\Omega)} \quad \text { and } \quad B(u, u) \geq C_{1}(\delta)\|u\|_{L^{2}(\Omega)}^{2} .
$$

We refer to [3, 5, 23, 29] for detailed analysis on the validity of (3.7) and (3.8) for various kernel functions. For instance, [23] established (3.7) and (3.8) for a number of different combinations of domains and constraints and for a kernel function $\gamma$ that is not only absolutely integrable but in fact, square integrable; in [29], the inequalities were shown for kernels that are absolutely integrable and uniformly bounded below by a positive constant everywhere; the case of nonnegative integrable kernels with compact support was studied in detail in [5] while the inequalities were also shown in [3] for a number of cases covered by the space equivalence results discussed in 7 .

With bilinear form (3.6), the problem (2.4) has the following weak formulation: find $u^{*} \in L_{n 0}^{2}(\Omega)$, such that for any $v \in L_{n 0}^{2}(\Omega)$,

$$
B(u, v)=(f, v),
$$

where $(f, v)=\int_{\Omega_{s}} f v d \mathbf{x}$.

For $u, v \in L_{n 0}^{2}(\Omega)$, we aim to decompose the bilinear form

$$
B(u, v)=\int_{\Omega} \int_{\Omega} \mathcal{D}^{*}(u) \beta \mathcal{D}^{*}(v) d \mathbf{x}^{\prime} d \mathbf{x}
$$


into the sum of local integrals over each element $K$ to get a nonlocal a posterior error estimator for the nonlocal diffusion equation (2.4).

3.2. Finite element discretization and a posteriori error estimate. To study the numerical solution of the linear nonlocal diffusion problem (2.4) by finite element methods, we let $\mathbf{S}^{h}$ be a finite dimensional subspace of $L_{n 0}^{2}(\Omega)$, and $\mathbf{S}^{h}$ consists of piecewise smooth functions over $\{K\}$, where $\{K\}$ denotes the mesh of $\Omega_{s}\left(\Omega_{s}=\bigcup K\right)$.

By Lemma 3.2 , we have for any $u, v \in L_{n 0}^{2}(\Omega)$,

$$
\begin{aligned}
B(u, v) & =\int_{\Omega} \int_{\Omega} \mathcal{D}^{*}(u) \beta \mathcal{D}^{*}(v) d \mathbf{x}^{\prime} d \mathbf{x} \\
& =\int_{\Omega} v(\mathbf{x}) \mathcal{D}\left(\beta \mathcal{D}^{*}(u)\right) d \mathbf{x} \\
& =\int_{\Omega_{s}} v(\mathbf{x}) \mathcal{D}\left(\beta \mathcal{D}^{*}(u)\right) d \mathbf{x} .
\end{aligned}
$$

Let $u^{h} \in \mathbf{S}^{h}$ be the Galerkin approximation of $u$ in $\mathbf{S}^{h}$ obtained using the weak form (3.9), then we have that

$$
B\left(u^{h}, v^{h}\right)=\left(f, v^{h}\right), \quad \forall v^{h} \in \mathbf{S}^{h} .
$$

By setting $e^{h}=u^{*}-u^{h}$ and using (3.10), we get for any $v \in L_{n 0}^{2}(\Omega)$,

$$
\begin{aligned}
B\left(e^{h}, v\right) & =B\left(u^{*}, v\right)-B\left(u^{h}, v\right) \\
& =\int_{\Omega_{s}} v(\mathbf{x})\left(f-\mathcal{D}\left(\beta \mathcal{D}^{*}\left(u^{h}(\mathbf{x})\right)\right)\right) d \mathbf{x} \\
& =\left(R^{h}, v\right),
\end{aligned}
$$

where

$$
R^{h}(\mathbf{x})=f(\mathbf{x})-\mathcal{D}\left(\beta \mathcal{D}^{*}\left(u^{h}(\mathbf{x})\right)\right), \quad \forall \mathbf{x} \in K,
$$

denotes the residual term.

Under assumption (2.9), for $v \in L_{n 0}^{2}(\Omega)$, define the energy norm as

$$
\|v\|_{E}=\sqrt{B(v, v)}
$$

since (3.8) implies the equivalence between $\|v\|_{E}$ and $\|v\|_{L^{2}(\Omega)}$, so we can define the dual energy norm as

$$
\|v\|_{E^{*}}=\sup _{w \in L_{n 0}^{2}(\Omega)} \frac{(v, w)}{\|w\|_{E}}
$$

and we have

Lemma 3.3. For $u^{*}, u^{h} \in L_{n 0}^{2}(\Omega)$, the energy norm of error $e^{h}$ equals the dual energy norm of the corresponding residual $R^{h}$, i.e.,

$$
\left\|e^{h}\right\|_{E}=\left\|R^{h}\right\|_{E^{*}}
$$

Proof. First, we have by Cauchy-Schwarz inequality that for any $v \in L_{n 0}^{2}(\Omega)$,

$$
\left(R^{h}, v\right)=B\left(e^{h}, v\right) \leq\left\|e^{h}\right\|_{E} \cdot\|v\|_{E},
$$

SO

$$
\left\|R^{h}\right\|_{E^{*}}=\sup _{v} \frac{\left(R^{h}, v\right)}{\|v\|_{E}} \leq\left\|e^{h}\right\|_{E}
$$


Second, since $e^{h} \in L_{n 0}^{2}(\Omega)$,

$$
\left\|R^{h}\right\|_{E^{*}}=\sup _{v} \frac{\left(R^{h}, v\right)}{\|v\|_{E}} \geq \frac{B\left(e^{h}, e^{h}\right)}{\left\|e^{h}\right\|_{E}}=\left\|e^{h}\right\|_{E},
$$

then the conclusion follows.

We then have the following theorem for the a posteriori error approximation

Theorem 3.4. Let

$$
\eta_{\delta}^{h}=\frac{\left\|R^{h}\right\|_{L^{2}\left(\Omega_{s}\right)}}{\sqrt{M(\delta)}}
$$

denote the a posteriori error estimator. For fixed $\delta$, it satisfies that

$$
\frac{1}{2} \eta_{\delta}^{h} \leq\left\|e^{h}\right\|_{E} \leq \sqrt{\frac{M(\delta)}{C_{1}(\delta)}} \eta_{\delta}^{h}
$$

where $M(\delta)$ and $C_{1}(\delta)$ are as in (3.8).

Proof. By (3.8), we have for any $v \in L_{n 0}^{2}(\Omega)$,

$$
\sqrt{C_{1}(\delta)}\|v\|_{L^{2}(\Omega)} \leq\|v\|_{E} \leq 2 \sqrt{M(\delta)}\|v\|_{L^{2}(\Omega)}
$$

using the definition of $\|\cdot\|_{E^{*}}$ and Cauchy-Schwarz inequality, we obtain

$$
\frac{1}{2 \sqrt{M(\delta)}}\|v\|_{L^{2}(\Omega)} \leq\|v\|_{E^{*}} \leq \frac{1}{\sqrt{C_{1}(\delta)}}\|v\|_{L^{2}(\Omega)} .
$$

Let $v=R^{h}$ in the above inequality, applying Lemma 3.3 gives the conclusion.

Remark 3.5. It is worth noting that in Theorem 3.4, we can provide $\left\|e^{h}\right\|_{E}$ on both the upper and lower bounds, up to constant factors, in terms of $\eta_{\delta}^{h}$. This is largely a consequence of the boundedness of the nonlocal diffusion operator and the solution operator in the energy space. It is interesting to examine if the constant factors appearing in the bounds are tight with respect to changes in the model parameters. The numerical experiments given later indicate that, for small $\delta$, the lower bound is tight, while the upper bound fails to give a tight bound. This is not surprising as in the limit $\delta \rightarrow 0$, the nonlocal problems have been shown to reduce to their local counterparts [22, 24, 40, and for classical differential equations, a posteriori estimators take on different forms.

3.3. Relation with the local case. For simplicity, we assume $\Omega_{c}$ is a layered strip, with thickness $\delta$, surrounding $\Omega_{s}$, see Figure 1. Since the solution of the

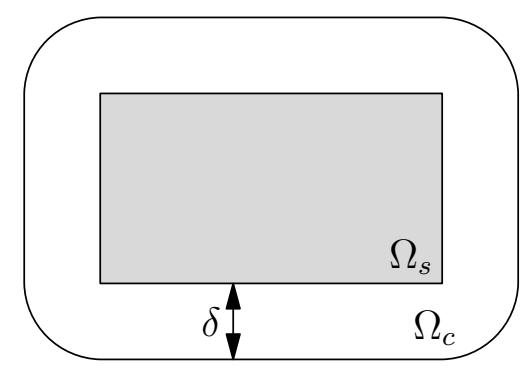

FiguRE $1 . \Omega_{s}$ and $\Omega_{c}$. 
problem (2.4) over $\Omega$ converges to the local limit, that is, the classic differential boundary value problem (2.10) over $\Omega_{s}$ as $\delta \rightarrow 0$ [22, 24, 40], with

$$
\mathbb{D}=\lim _{\delta \rightarrow 0} \mathbb{D}_{\delta}
$$

where $\left(\mathbb{D}_{\delta}\right)_{i j}=\int_{B_{\delta}(0)} \gamma z_{i} z_{j} d \mathbf{z}$ for $i, j=1,2, \ldots, d, \mathbf{z}=\left(z_{1}, z_{2}, \ldots, z_{d}\right)^{T} \in \mathbb{R}^{d}$. It is thus natural to study if the a posteriori error estimator derived earlier for a nonlocal problem would converge to the corresponding estimator of its local limit in some suitable sense.

To study the limiting behavior as $\delta \rightarrow 0$ of the a posterior error estimator on a given mesh, we assume that $\delta$ is sufficiently small such that $\delta \ll h_{K}$, in the remaining discussion provided in this section. For the finite element analysis carried out here, we focus on the case of having globally continuous and piecewise smooth basis functions so that a conforming Galerkin approximation of the PDE model is given in the limit with the corresponding a posteriori error estimation briefly reviewed in Section 2.4.

Remark 3.6. Notice that a virtue of nonlocal or PD models is to handle discontinuous solutions, due to which we can use discontinuous finite element basis functions without any change to the weak formulation (3.9). Since the local a posteriori estimators with continuous and discontinuous basis functions are essentially different, so we would start the discussion of the limit case from continuous finite element. More general analysis involving those based on discontinuous basis functions will be studied in the future.

To balance the generality of analysis and the simplicity of notation, we further assume that, in this subsection, $u$ is defined over the tessellation $\{K\}$ such that

$$
u \in C^{0}(\Omega) \cap H^{1}(\Omega),\left.\quad u\right|_{\Omega_{c}}=0, \quad u_{K} \in C^{\tau}(K) \text { for any } K,
$$

where $\tau \geq 2, u_{\chi}(\cdot)$ denotes the restriction of $u(\cdot)$ over any set $\chi$. We impose the same assumption on $v$. Be aware that $u$ does not need to solve weak form (3.9).

For any $K \subset \Omega, \Gamma_{K}$ is defined by

$$
\Gamma_{K}:=\{\mathbf{x} \notin K \mid \operatorname{Dist}(\mathbf{x}, K) \leq \delta\},
$$

then it follows that

$$
\begin{gathered}
-\int_{K} v(\mathbf{x}) \mathcal{D}\left(\beta \mathcal{D}^{*}(u)\right) d \mathbf{x}=\int_{K} v(\mathbf{x}) \int_{K} 2 \gamma \cdot\left(u\left(\mathbf{x}^{\prime}\right)-u(\mathbf{x})\right) d \mathbf{x}^{\prime} d \mathbf{x} \\
+\int_{K} v(\mathbf{x}) \int_{\Gamma_{K}} 2 \gamma \cdot\left(u\left(\mathbf{x}^{\prime}\right)-u(\mathbf{x})\right) d \mathbf{x}^{\prime} d \mathbf{x} .
\end{gathered}
$$

Denote $K=K_{\text {in }} \cup K_{\text {out }}$, where $K_{\text {out }}=K \cap B_{\delta}(\partial K)$ (the $\delta$-neighborhood of $\partial K$ ), while $K_{\text {in }}=K / K_{\text {out }}$ (see Figure 2 for an illustration of the two-dimensional case). When $\mathbf{x}^{\prime}, \mathbf{x} \in K$, since $u$ is smooth within an element $K$, by Taylor expansion, we get

$$
u\left(\mathbf{x}^{\prime}\right)=u(\mathbf{x})+\nabla u(\mathbf{x}) \cdot\left(\mathbf{x}^{\prime}-\mathbf{x}\right)+\frac{1}{2}\left(\mathbf{x}^{\prime}-\mathbf{x}\right)^{T} H_{u}(\mathbf{x})\left(\mathbf{x}^{\prime}-\mathbf{x}\right)+o\left(\delta^{2}\right),
$$

where $H_{u}$ denotes the Hessian of $u$. Then the first term in (3.21) becomes 


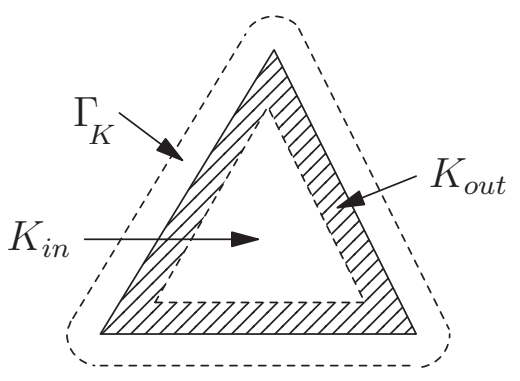

Figure 2. $K=K_{\text {in }} \cup K_{\text {out }}$, an illustration in the two-dimensional space.

$$
\begin{aligned}
\int_{K} v(\mathbf{x}) & \int_{K} 2 \gamma \cdot\left(u\left(\mathbf{x}^{\prime}\right)-u(\mathbf{x})\right) d \mathbf{x}^{\prime} d \mathbf{x} \\
= & \int_{K} v(\mathbf{x}) \nabla u(\mathbf{x}) \cdot \int_{K} 2 \gamma \cdot\left(\mathbf{x}^{\prime}-\mathbf{x}\right) d \mathbf{x}^{\prime} d \mathbf{x} \\
& +\int_{K} v(\mathbf{x}) \int_{K} \gamma \cdot\left(\mathbf{x}^{\prime}-\mathbf{x}\right)^{T} H_{u}(\mathbf{x})\left(\mathbf{x}^{\prime}-\mathbf{x}\right) d \mathbf{x}^{\prime} d \mathbf{x} \\
& +\int_{K} v(\mathbf{x}) \int_{K} 2 \gamma \cdot o\left(\delta^{2}\right) d \mathbf{x}^{\prime} d \mathbf{x} \\
= & T_{1}+T_{2}+T_{3} .
\end{aligned}
$$

Since $u$ is piecewise smooth for $\mathbf{x} \in K$ and $\mathbf{x}^{\prime} \in \Gamma_{K}$, respectively, we define $\mathbf{s}$ as the intersection of $\partial K$ and the vector $\left(\mathbf{x}^{\prime}-\mathbf{x}\right)$ (as shown in Figure 4), one then has the following Taylor expansion

$$
u_{\Gamma_{K}}\left(\mathbf{x}^{\prime}\right)=u(\mathbf{x})+\nabla u_{K}(\mathbf{x}) \cdot(\mathbf{s}-\mathbf{x})+\nabla u_{\Gamma_{K}}(\mathbf{s}) \cdot\left(\mathbf{x}^{\prime}-\mathbf{s}\right)+o(\delta) .
$$

Then, the second term in (3.21) can be computed as follows:

$$
\begin{aligned}
\int_{K} v(\mathbf{x}) & \int_{\Gamma_{K}} 2 \gamma \cdot\left(u\left(\mathbf{x}^{\prime}\right)-u(\mathbf{x})\right) d \mathbf{x}^{\prime} d \mathbf{x} \\
= & \int_{K_{\text {out }}} v(\mathbf{x}) \int_{\Gamma_{K}} 2 \gamma \cdot\left(\nabla u_{K}(\mathbf{x}) \cdot(\mathbf{s}-\mathbf{x})+\nabla u_{\Gamma_{K}}(\mathbf{s}) \cdot\left(\mathbf{x}^{\prime}-\mathbf{s}\right)\right) d \mathbf{x}^{\prime} d \mathbf{x} \\
& +\int_{K_{\text {out }}} v(\mathbf{x}) \int_{\Gamma_{K}} 2 \gamma \cdot o(\delta) d \mathbf{x}^{\prime} d \mathbf{x} \\
= & \int_{K_{\text {out }}} v(\mathbf{x}) \nabla u_{K}(\mathbf{x}) \cdot \int_{\Gamma_{K}} 2 \gamma \cdot\left(\mathbf{x}^{\prime}-\mathbf{x}\right) d \mathbf{x}^{\prime} d \mathbf{x} \\
& +\int_{K_{\text {out }}} v(\mathbf{x}) \int_{\Gamma_{K}} \nabla u_{K}(\mathbf{x}) \cdot 2 \gamma \cdot\left(\mathbf{s}-\mathbf{x}^{\prime}\right) d \mathbf{x}^{\prime} d \mathbf{x} \\
& +\int_{K_{\text {out }}} v(\mathbf{x}) \int_{\Gamma_{K}} \nabla u_{\Gamma_{K}}(\mathbf{s}) \cdot 2 \gamma \cdot\left(\mathbf{x}^{\prime}-\mathbf{s}\right) d \mathbf{x}^{\prime} d \mathbf{x} \\
& +\int_{K_{\text {out }}} v(\mathbf{x}) \int_{\Gamma_{K}} 2 \gamma \cdot o(\delta) d \mathbf{x}^{\prime} d \mathbf{x} \\
= & \int_{K_{\text {out }}} v(\mathbf{x}) \nabla u_{K}(\mathbf{x}) \cdot \int_{\Gamma_{K}} 2 \gamma \cdot\left(\mathbf{x}^{\prime}-\mathbf{x}\right) d \mathbf{x}^{\prime} d \mathbf{x} \\
& +\int_{K_{\text {out }}} v(\mathbf{x}) \int_{\Gamma_{K}}\left(\nabla u_{\Gamma_{K}}(\mathbf{s})-\nabla u_{K}(\mathbf{x})\right) \cdot 2 \gamma \cdot\left(\mathbf{x}^{\prime}-\mathbf{s}\right) d \mathbf{x}^{\prime} d \mathbf{x}
\end{aligned}
$$




$$
\begin{aligned}
& +\int_{K_{\text {out }}} v(\mathbf{x}) \int_{\Gamma_{K}} 2 \gamma \cdot o(\delta) d \mathbf{x}^{\prime} d \mathbf{x} \\
= & S_{1}+S_{2}+S_{3} .
\end{aligned}
$$

Next we will try to evaluate $\left\{T_{i}, S_{i}\right\}, i=1,2,3$. First, by the symmetry of $\gamma$, we get

$$
\begin{aligned}
T_{1}+S_{1} & =\int_{K} v(\mathbf{x}) \nabla u_{K}(\mathbf{x}) \cdot \int_{K \cup \Gamma_{K}} 2 \gamma \cdot\left(\mathbf{x}^{\prime}-\mathbf{x}\right) d \mathbf{x}^{\prime} d \mathbf{x} \\
& =\int_{K} v(\mathbf{x}) \nabla u_{K}(\mathbf{x}) \cdot \int_{B_{\delta}(\mathbf{x})} 2 \gamma \cdot\left(\mathbf{x}^{\prime}-\mathbf{x}\right) d \mathbf{x}^{\prime} d \mathbf{x} \\
& =0 .
\end{aligned}
$$

It is also easy to see that with assumption (2.9),

$$
T_{3} \rightarrow 0, \quad S_{3} \rightarrow 0 \quad \text { as } \quad \delta \rightarrow 0 .
$$

It now only remains to evaluate $T_{2}$ and $S_{2}$. Define

$$
\widehat{T}_{2}=\int_{K_{\text {out }}} v(\mathbf{x}) \int_{K} \gamma \cdot\left(\mathbf{x}^{\prime}-\mathbf{x}\right)^{T} H_{u}(\mathbf{x})\left(\mathbf{x}^{\prime}-\mathbf{x}\right) d \mathbf{x}^{\prime} d \mathbf{x}
$$

and it follows that

$$
\widehat{T}_{2} \rightarrow 0 \quad \text { as } \quad \delta \rightarrow 0 .
$$

Let us substitute $\left(\mathbf{x}^{\prime}-\mathbf{x}\right)$ by $\mathbf{z}=\left(z_{1}, z_{2}, \ldots, z_{d}\right)^{T}$, then

$$
\begin{aligned}
T_{2} & =\int_{K_{i n}} v(\mathbf{x}) \int_{K} \gamma \cdot\left(\mathbf{x}^{\prime}-\mathbf{x}\right)^{T} H_{u}(\mathbf{x})\left(\mathbf{x}^{\prime}-\mathbf{x}\right) d \mathbf{x}^{\prime} d \mathbf{x}+\widehat{T}_{2} \\
& =\int_{K_{i n}} v(\mathbf{x}) \int_{B_{\delta}(0)} \gamma \cdot \mathbf{z}^{T} H_{u}(\mathbf{x}) \mathbf{z} d \mathbf{z} d \mathbf{x}+\widehat{T}_{2} \\
& =\int_{K_{i n}} v(\mathbf{x}) \int_{B_{\delta}(0)} \gamma \cdot\left(\sum_{i, j} z_{i} z_{j} u_{i j}(\mathbf{x})\right) d \mathbf{z} d \mathbf{x}+\widehat{T}_{2} \\
& =\int_{K_{i n}} \nabla \cdot\left(\mathbb{D}_{\delta} \nabla u(\mathbf{x})\right) v(\mathbf{x}) d \mathbf{x}+\widehat{T}_{2},
\end{aligned}
$$

where $u_{i j}$ denotes the partial derivatives of $u$ along $x_{i}$ and $x_{j}$ directions, respectively, $\mathbb{D}_{\delta}$ is an $d \times d$ symmetric matrix defined by

$$
\left(\mathbb{D}_{\delta}\right)_{i j}=\int_{B_{\delta}(0)} \gamma z_{i} z_{j} d \mathbf{z}
$$

Finally,

$$
S_{2}=\int_{K_{\text {out }}} v(\mathbf{x}) \int_{\Gamma_{K}} 2 \gamma\left(\nabla u_{\Gamma_{K}}(\mathbf{s})-\nabla u_{K}(\mathbf{x})\right) \cdot\left(\mathbf{x}^{\prime}-\mathbf{s}\right) d \mathbf{x}^{\prime} d \mathbf{x} .
$$

For the convenience of later computations, since $\delta$ is sufficiently small we rewrite the above equation as

$$
\begin{aligned}
S_{2}= & \sum_{\mathbf{r} \in \operatorname{edge}(K)} \int_{K_{\text {out }} \cap B_{\delta}(\mathbf{r})} v(\mathbf{x}) \int_{\Gamma_{K} \cap B_{\delta}(\mathbf{r})} 2 \gamma \\
& \cdot\left(\nabla u_{\Gamma_{K}}(\mathbf{s})-\nabla u_{K}(\mathbf{x})\right) \cdot\left(\mathbf{x}^{\prime}-\mathbf{s}\right) d \mathbf{x}^{\prime} d \mathbf{x}+o(1),
\end{aligned}
$$

where edge $(K)$ denotes the set of boundary edges (faces) of $K$ and $B_{\delta}(\mathbf{r})$ the $\delta$-neighborhood of edge (face) $\mathbf{r}$. 


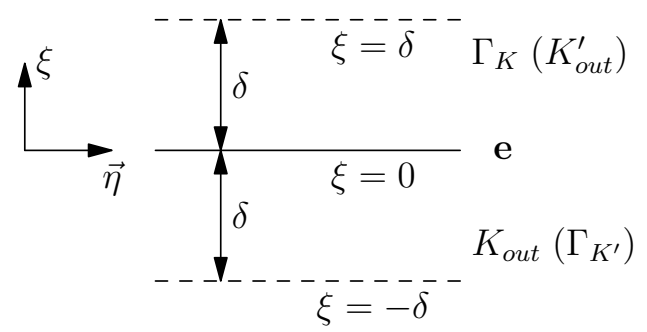

FiguRE 3. The $\delta$-neighborhood of an edge e.

Suppose that elements $K$ and $K^{\prime}$ have a common edge (face) e. If we repeat the above computation on $K^{\prime}$, we can get the terms $T_{1}^{\prime}, T_{2}^{\prime}, T_{3}^{\prime}, S_{1}^{\prime}, S_{2}^{\prime}$ and $S_{3}^{\prime}$ corresponding to $T_{1}, T_{2}, T_{3}, S_{1}, S_{2}$ and $S_{3}$, respectively, among which

$$
\begin{aligned}
S_{2}^{\prime}= & \sum_{\mathbf{r} \in \operatorname{edge}\left(K^{\prime}\right)} \int_{K_{\text {out }}^{\prime} \cap B_{\delta}(\mathbf{r})} v(\mathbf{x}) \int_{\Gamma_{K^{\prime}} \cap B_{\delta}(\mathbf{r})} 2 \gamma \\
& \cdot\left(\nabla u_{\Gamma_{K^{\prime}}}(\mathbf{s})-\nabla u_{K^{\prime}}(\mathbf{x})\right) \cdot\left(\mathbf{x}^{\prime}-\mathbf{s}\right) d \mathbf{x}^{\prime} d \mathbf{x}+o(1) .
\end{aligned}
$$

Without loss of generality, assume the outward normal of $K$ on $\mathbf{e}$ is $\overrightarrow{\mathbf{n}}_{\mathbf{e}}=$ $(1,0,0, \ldots, 0)^{T} \in \mathbb{R}^{d}$. Here we use the coordinate system $\xi \times \vec{\eta}$, where $\xi \in \mathbb{R}$ and $\vec{\eta} \in \mathbb{R}^{d-1}$, as shown in Figure 3, i.e., edge (or face) e is on the $(d-1)$-dimensional plane $\xi=0$.

One can easily observe that

$$
(\mathbf{e} \times[-\delta, 0]) \approx K_{\text {out }} \cap B_{\delta}(\mathbf{e}) \approx \Gamma_{K^{\prime}} \cap B_{\delta}(\mathbf{e}),
$$

the difference between any two of the above sets is of magnitude $O\left(\delta^{d}\right)$, which means that the difference can be ignored in limit sense. Similarly, we can also have

$$
(\mathbf{e} \times[0, \delta]) \approx K_{\text {out }}^{\prime} \cap B_{\delta}(\mathbf{e}) \approx \Gamma_{K} \cap B_{\delta}(\mathbf{e}),
$$

where the difference between any two sets again can be ignored in limit sense.

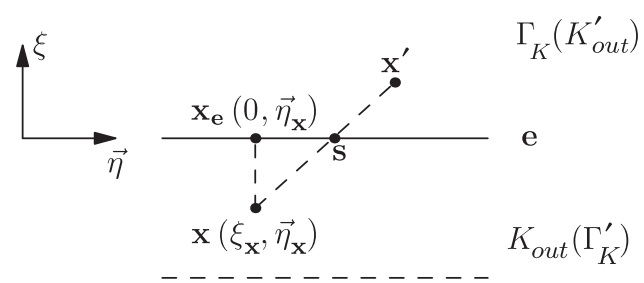

Figure 4. A projection of $\mathbf{x}$ onto $\mathbf{e}$.

Then let us add (3.28) and (3.29), and evaluate the contribution from edge (or face) e as follows (drop the high order terms):

$$
\begin{aligned}
& \int_{K_{\text {out }} \cap B_{\delta}(\mathbf{e})} v(\mathbf{x}) \int_{\Gamma_{K} \cap B_{\delta}(\mathbf{e})} 2 \gamma \cdot\left(\nabla u_{\Gamma_{K}}(\mathbf{s})-\nabla u_{K}(\mathbf{x})\right) \cdot\left(\mathbf{x}^{\prime}-\mathbf{s}\right) d \mathbf{x}^{\prime} d \mathbf{x} \\
& +\int_{K_{\text {out }}^{\prime} \cap B_{\delta}(\mathbf{e})} v(\mathbf{x}) \int_{\Gamma_{K^{\prime}} \cap B_{\delta}(\mathbf{e})} 2 \gamma \cdot\left(\nabla u_{\Gamma_{K^{\prime}}}(\mathbf{s})-\nabla u_{K^{\prime}}(\mathbf{x})\right) \cdot\left(\mathbf{x}^{\prime}-\mathbf{s}\right) d \mathbf{x}^{\prime} d \mathbf{x}
\end{aligned}
$$




$$
\begin{aligned}
= & \int_{K_{\text {out }} \cap B_{\delta}(\mathbf{e})} v(\mathbf{x}) \int_{\Gamma_{K} \cap B_{\delta}(\mathbf{e})} 2 \gamma \cdot\left(\nabla u_{\Gamma_{K}}(\mathbf{s})-\nabla u_{K}(\mathbf{x})\right) \cdot\left(\mathbf{x}^{\prime}-\mathbf{s}\right) d \mathbf{x}^{\prime} d \mathbf{x} \\
& +\int_{\Gamma_{K^{\prime}} \cap B_{\delta}(\mathbf{e})} \int_{K_{\text {out }}^{\prime} \cap B_{\delta}(\mathbf{e})} 2 \gamma \cdot v\left(\mathbf{x}^{\prime}\right)\left(\nabla u_{\Gamma_{K^{\prime}}}(\mathbf{s})-\nabla u_{K^{\prime}}\left(\mathbf{x}^{\prime}\right)\right) \cdot(\mathbf{x}-\mathbf{s}) d \mathbf{x}^{\prime} d \mathbf{x} \\
= & \int_{\mathbf{e}} \int_{-\delta}^{0} \int_{B_{\delta}(\mathbf{x}) \cap \Gamma_{K}} 2 \gamma \cdot v(\mathbf{x})\left(\nabla u_{\Gamma_{K}}(\mathbf{s})-\nabla u_{K}(\mathbf{x})\right) \cdot\left(\mathbf{x}^{\prime}-\mathbf{s}\right) d \mathbf{x}^{\prime} d \xi d \vec{\eta} \\
& +\int_{\mathbf{e}} \int_{-\delta}^{0} \int_{B_{\delta}(\mathbf{x}) \cap K_{\text {out }}^{\prime}} 2 \gamma \cdot v\left(\mathbf{x}^{\prime}\right)\left(\nabla u_{K^{\prime}}\left(\mathbf{x}^{\prime}\right)-\nabla u_{\Gamma_{K^{\prime}}}(\mathbf{s})\right) \cdot(\mathbf{s}-\mathbf{x}) d \mathbf{x}^{\prime} d \xi d \vec{\eta},
\end{aligned}
$$

where the second to the last step is obtained by switching order of integration; the last step is obtained by using (3.30) and the fact that $\mathbf{x}^{\prime}$ interacts with $\mathbf{x}$ only if $\mathbf{x}^{\prime} \in B_{\delta}(\mathbf{x})$. Note that in the above derivation we let $\mathbf{x}=\left(\xi_{\mathbf{x}}, \vec{\eta}_{\mathbf{x}}\right)$, substitute $K_{\text {out }}^{\prime}$ by $\Gamma_{K}, u_{K^{\prime}}$ by $u_{\Gamma_{K}}, u_{\Gamma_{K^{\prime}}}$ by $u_{K}$ in (3.32), and denote by $\mathbf{x}_{\mathbf{e}}=\left(0, \vec{\eta}_{\mathbf{x}}\right)$ the projection of $\mathbf{x}$ on $\mathbf{e}$, as shown in Figure 4 .

Since $\mathbf{x}, \mathbf{x}^{\prime}$ and $\mathbf{s}$ are all in the $\delta$-neighborhood of $\mathbf{x}_{\mathbf{e}}$, we can perform the Taylor expansion for $v(\mathbf{x}), v\left(\mathbf{x}^{\prime}\right), \nabla u_{\Gamma_{K}}(\mathbf{s}), \nabla u_{\Gamma_{K}}\left(\mathbf{x}^{\prime}\right), \nabla u_{K}(\mathbf{x})$ and $\nabla u_{K}(\mathbf{s})$ at point $\mathbf{x}_{\mathbf{e}}$, respectively, it follows that (3.32) can be approximated as, after dropping the high order terms,

$$
\begin{gathered}
\int_{\mathbf{e}} v\left(\mathbf{x}_{\mathbf{e}}\right)\left(\nabla u_{\Gamma_{K}}\left(\mathbf{x}_{\mathbf{e}}\right)-\nabla u_{K}\left(\mathbf{x}_{\mathbf{e}}\right)\right) \int_{-\delta}^{0} \int_{B_{\delta}(\mathbf{x}) \cap \Gamma_{K}} 2 \gamma \cdot\left(\mathbf{x}^{\prime}-\mathbf{x}\right) d \mathbf{x}^{\prime} d \xi d \vec{\eta} \\
=\int_{\mathbf{e}} v\left(\mathbf{x}_{\mathbf{e}}\right)\left(\nabla u_{\Gamma_{K}}\left(\mathbf{x}_{\mathbf{e}}\right)-\nabla u_{K}\left(\mathbf{x}_{\mathbf{e}}\right)\right) \int_{-\delta}^{0} \int_{S C(\delta, \delta+\xi)} 2 \gamma \mathbf{z} d \mathbf{z} d \xi d \vec{\eta},
\end{gathered}
$$

where $\mathbf{z}=\mathbf{x}^{\prime}-\mathbf{x}$, the integral domain $S C(\delta, \delta+\xi)$ denotes the spherical cap of a sphere with radius $\delta$ and height $\delta+\xi$, as shown in Figure 5 .

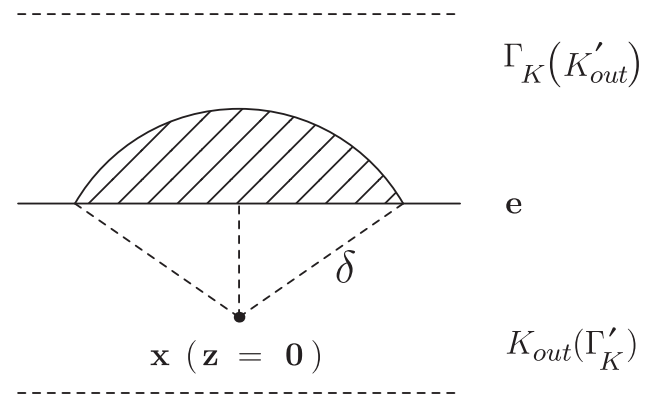

FiguRE 5. An illustration of the integral domain $S C(\delta, \delta+\xi)$ that denotes the spherical cap of a sphere with radius $\delta$ and height $\delta+\xi$.

From (3.33) we obtain the jump of $\nabla u$ on interior edge $\mathbf{e}$, and it remains to show that the integral

$$
\int_{-\delta}^{0} \int_{S C(\delta, \delta+\xi)} 2 \gamma \mathbf{z} d \mathbf{z} d \xi
$$

actually converges to outward normal with the same coefficient as the Laplacian term in (3.26). 
Let $\mathbf{z}=\left(z_{1}, z_{2}, \ldots, z_{d}\right)^{T}$, we switch the order of the integration, where we omit some details, in (3.34) and obtain the following result for fixed $\vec{\eta}_{\mathbf{x}}$,

$$
\begin{aligned}
\int_{-\delta}^{0} \int_{S C(\delta, \delta+\xi)} 2 \gamma \mathbf{z} d \mathbf{z} d \xi & =\int_{S C(\delta, \delta)} \int_{-z_{1}}^{0} 2 \gamma \mathbf{z} d \xi d \mathbf{z} \\
& =\int_{S C(\delta, \delta)} 2 \gamma z_{1} \mathbf{z} d \mathbf{z} \\
& =\int_{S C(\delta, \delta)} 2 \gamma \cdot\left(z_{1}^{2}, z_{1} z_{2}, z_{1} z_{3}, \ldots, z_{1} z_{d}\right)^{T} d \mathbf{z} \\
& =\int_{B_{\delta}(0)} \gamma \cdot\left(z_{1}^{2}, z_{1} z_{2}, z_{1} z_{3}, \ldots, z_{1} z_{d}\right)^{T} d \mathbf{z} \\
& =\mathbb{D}_{\delta} \cdot \overrightarrow{\mathbf{n}}_{\mathbf{e}},
\end{aligned}
$$

where the second to last equality holds since the integration domain $S C(\delta, \delta)$ is actually a half-sphere.

When $\mathbf{e}$ is not horizontal, we can simply perform a rotation operator on $\mathbf{x}^{\prime}$ and $\mathbf{x}$, then a similar result as in 3.35 follows.

Now we go back to (3.32), it is easy to see that

$$
\begin{aligned}
\int_{K_{\text {out }} \cap B_{\delta}(\mathbf{e})} v(\mathbf{x}) \int_{\Gamma_{K} \cap B_{\delta}(\mathbf{e})} 2 \gamma \cdot\left(\nabla u_{\Gamma_{K}}(\mathbf{s})-\nabla u_{K}(\mathbf{x})\right) \cdot\left(\mathbf{x}^{\prime}-\mathbf{s}\right) d \mathbf{x}^{\prime} d \mathbf{x}+o(\delta) \\
=\int_{K_{\text {out }}^{\prime} \cap B_{\delta}(\mathbf{e})} v(\mathbf{x}) \int_{\Gamma_{K^{\prime}} \cap B_{\delta}(\mathbf{e})} 2 \gamma \cdot\left(\nabla u_{\Gamma_{K^{\prime}}}(\mathbf{s})-\nabla u_{K^{\prime}}(\mathbf{x})\right) \cdot\left(\mathbf{x}^{\prime}-\mathbf{s}\right) d \mathbf{x}^{\prime} d \mathbf{x}
\end{aligned}
$$

and the limit of each integral exists as $\delta \rightarrow 0$.

Considering (3.28), (3.32), (3.33), (3.35) and (3.36), we have that as $\delta \rightarrow 0$,

$$
S_{2} \rightarrow-\frac{1}{2} \sum_{\mathbf{r} \in e d g e(K)} \int_{\mathbf{r}} v(\nu) \llbracket \mathbb{D} \nabla u(\nu) \rrbracket_{\mathbf{r}} d \nu,
$$

where $\mathbb{D}=\lim _{\delta \rightarrow 0} \mathbb{D}_{\delta}, \llbracket \mathbb{D} \nabla u(\cdot) \rrbracket_{\mathbf{r}}$ denotes the jump of $\mathbb{D} \nabla u$ on $\mathbf{r}$.

Combining (3.21), (3.22), (3.23), (3.24), (3.25), (3.26) and (3.37), we obtain the following theorem.

Theorem 3.7. Under assumption (2.9), for $u$ and $v$ that satisfy assumption (3.19), as $\delta \rightarrow 0$ on any element $K$, we get

$$
\begin{aligned}
&-\int_{K} v(\mathbf{x}) \mathcal{D}\left(\beta \mathcal{D}^{*}(u)\right) d \mathbf{x} \\
& \rightarrow \int_{K} v(\mathbf{x})(\nabla \cdot(\mathbb{D} \nabla u(\mathbf{x}))) d \mathbf{x}-\frac{1}{2} \int_{\partial K} v(\nu) \llbracket \mathbb{D} \nabla u(\nu) \rrbracket_{\mathbf{r}} d \nu .
\end{aligned}
$$

Consequently, we have

Corollary 3.8. Under assumption (2.9), for $u^{h}$ and $v$ that satisfy assumption (3.19), as $\delta \rightarrow 0$ on any element $K$,

$$
\begin{aligned}
& \int_{K} v(\mathbf{x})\left(f(\mathbf{x})-\mathcal{D}\left(\beta \mathcal{D}^{*}\left(u^{h}\right)\right)\right) d \mathbf{x} \\
& \quad \rightarrow \int_{K} v(\mathbf{x})\left(f(\mathbf{x})+\nabla \cdot\left(\mathbb{D} \nabla u^{h}(\mathbf{x})\right)\right) d \mathbf{x}-\frac{1}{2} \int_{\partial K} v(\nu) \llbracket \mathbb{D} \nabla u^{h}(\nu) \rrbracket_{\mathbf{r}} d \nu
\end{aligned}
$$


that is,

$$
\int_{K} v(\mathbf{x}) R^{h} d \mathbf{x} \rightarrow \int_{K} v(\mathbf{x}) r_{*}^{h} d \mathbf{x}-\frac{1}{2} \int_{\partial K} v(\nu) R_{*}^{h} d \nu .
$$

Remark 3.9. Notice that in Theorem 3.7. we actually proved the local limit of nonlocal operator on each element. Also, comparing (3.39) with (2.12), as $\delta \rightarrow 0$, the nonlocal residual-based a posteriori error approximation is consistent with the classical a posteriori error approximation (in the weak sense).

Summing up (3.38) for all the $K$ 's, we would have as $\delta \rightarrow 0$,

$$
\begin{aligned}
& -\int_{\Omega} \int_{\Omega} \mathcal{D}^{*}(v) \beta \mathcal{D}^{*}(u) d \mathbf{x}^{\prime} d \mathbf{x}=-\int_{\Omega_{s}} v(\mathbf{x}) \mathcal{D}\left(\beta \mathcal{D}^{*}(u)\right) d \mathbf{x} \\
& \quad \rightarrow \sum_{K}\left(\int_{K} v(\mathbf{x})(\nabla \cdot(\mathbb{D} \nabla u(\mathbf{x}))) d \mathbf{x}-\frac{1}{2} \int_{\partial K} v(\nu) \llbracket \mathbb{D} \nabla u(\nu) \rrbracket_{\mathbf{r}} d \nu\right) \\
& =-\int_{\Omega_{s}} \nabla u(\mathbf{x}) \cdot \mathbb{D} \cdot \nabla v(\mathbf{x}) d \mathbf{x},
\end{aligned}
$$

which implies the nonlocal diffusion constraint value problem reduces to classical Poisson equation with Dirichlet boundary condition, as stated in [22, 29].

Remark 3.10. We have proved the weak convergence of the nonlocal a posteriori error estimator to its local counterpart, while it is more interesting and challenging to establish the connection between the nonlocal and local estimators in a strong sense. By using the results in 15, 29, it is not difficult to prove the convergence of the nonlocal energy norm, as defined in (3.14) , to $H^{1}$ norm as $\delta \rightarrow 0$. Therefore, if the error estimator $\eta_{\delta}^{h}$ approximates $\left\|e^{h}\right\|_{E}$ robustly (independent of the choice of $\delta$ ), its limit should approximate $\left\|e^{h}\right\|_{H^{1}}$ (if the norm is well defined), which implies that the nonlocal estimator converges to the local estimator for the corresponding PDE. Although we have not proved such independence in this paper, this conclusion could be observed from the various numerical experiments we will demonstrate in Section 5

\section{A POSTERIORI ERROR ANALYSIS OF PERIDYNAMIC EQUATIONS}

Under the assumption (2.9), we define the variational form of problem (2.8): find $\mathbf{u}^{*} \in \mathbf{L}_{n 0}^{2}(\Omega)=\left(L_{n 0}^{2}(\Omega)\right)^{d}$, such that for any $\mathbf{v} \in \mathbf{L}_{n 0}^{2}(\Omega)$,

$$
\mathbf{B}\left(\mathbf{u}^{*}, \mathbf{v}\right)=(\mathbf{f}, \mathbf{v})
$$

where the bilinear form is given by

$$
\mathbf{B}(\mathbf{u}, \mathbf{v})=\int_{\Omega} \int_{\Omega} \mathcal{G}^{*}(\mathbf{u}) \beta \mathcal{G}^{*}(\mathbf{v}) d \mathbf{x}^{\prime} d \mathbf{x}
$$

and

$$
(\mathbf{f}, \mathbf{v})=\int_{\Omega_{s}} \mathbf{f} \cdot \mathbf{v} d \mathbf{x}
$$

For the simplicity of analysis, we study the isotropic and homogeneous kernel function instead of the more general anisotropic kernels considered earlier in the case of nonlocal diffusion equations, so that we may avoid complicated notations. We note that there is no essential difference in the final conclusions between the 
isotropic and anisotropic cases. Thus, let us assume that $\beta$ is isotropic and homogeneous, that is, a function of $\left|\mathbf{x}^{\prime}-\mathbf{x}\right|$. Moreover, for a fixed $\delta>0$,

$$
\beta\left(\mathbf{x}^{\prime}, \mathbf{x}\right)=\left\{\begin{array}{lr}
\beta\left(\left|\mathbf{x}^{\prime}-\mathbf{x}\right|\right) & \text { when }\left|\mathbf{x}^{\prime}-\mathbf{x}\right|<\delta \\
0 & \text { otherwise. }
\end{array}\right.
$$

We set

$$
\boldsymbol{\alpha}\left(\mathrm{x}^{\prime}, \mathbf{x}\right)=\frac{\mathbf{x}^{\prime}-\mathbf{x}}{\left|\mathbf{x}^{\prime}-\mathbf{x}\right|}
$$

and define the kernel function for PD model with $\mathbf{z}=\mathbf{x}^{\prime}-\mathbf{x}$,

$$
\Lambda(\mathbf{z})=(\boldsymbol{\alpha} \otimes \boldsymbol{\alpha} \beta)(\mathbf{z})=\frac{\mathbf{z} \otimes \mathbf{z}}{|\mathbf{z}|^{2}} \beta(|\mathbf{z}|),
$$

which is also called the micromodulus function [25, 34].

With the above $\boldsymbol{\alpha}$ and $\beta$, we can write (2.9) as

$$
0<c(\delta) \leq \int_{B_{\delta}(0)} \beta(|\mathbf{z}|) d \mathbf{z} \leq M(\delta)<\infty .
$$

In [24, the well-posedness of linear state-based and bond-based PD models with solutions in $\mathbf{L}^{2}(\Omega)=\left(L^{2}(\Omega)\right)^{d}$ has been established for general symmetric square integrable kernel functions by showing the corresponding nonlocal bilinear form being continuous and coercive in the volume constrained space $\left(L_{n 0}^{2}(\Omega)\right)^{d}$. The coercivity is a particular consequence of spectral properties of the Hilbert-Schmidt operator and the precise characterization of the energy space. These results generalized similar properties proved under more special nonlocal boundary conditions in [40] with isotropic and homogeneous $L^{1}$ kernel functions. For the constrained volume condition considered here, similar to [3, 7, it is also possible to establish similar properties for the bilinear form with other kernels, say those homogeneous, isotropic and $L^{1}$ integrable kernels. In this paper, we focus on the finite element approximation by assuming the continuity and coercivity in the constrained energy space $\left(L_{n 0}^{2}(\Omega)\right)^{d}$ of the bilinear form corresponding to the linear bond-based PD model. That is, for any $\mathbf{u}, \mathbf{v} \in \mathbf{L}_{n 0}^{2}(\Omega)$, we assume that there exist nonnegative constants $C_{2}$ and $C_{3}(\delta)$ such that

$$
|\mathbf{B}(\mathbf{u}, \mathbf{v})| \leq C_{2} M(\delta)\|\mathbf{u}\|_{\mathbf{L}^{2}(\Omega)}\|\mathbf{v}\|_{\mathbf{L}^{2}(\Omega)} \text { and } \mathbf{B}(\mathbf{u}, \mathbf{v}) \geq C_{3}(\delta)\|\mathbf{u}\|_{\mathbf{L}^{2}(\Omega)}^{2} .
$$

Then, similar to the discussion earlier, we may state the following lemma about nonlocal Green's identity,

Lemma 4.1. Assume the kernel function $\Lambda$ be defined as in (4.2), with $\boldsymbol{\alpha}$ and $\beta$ satisfying (2.9). Then for any $\mathbf{u}, \mathbf{v} \in \mathbf{L}_{n 0}^{2}(\Omega)$,

$$
\int_{\Omega_{s}} \mathbf{v} \mathcal{G}\left(\beta \mathcal{G}^{*}(\mathbf{u})\right) d \mathbf{x}-\int_{\Omega} \int_{\Omega} \mathcal{G}^{*}(\mathbf{v}) \beta \mathcal{G}^{*}(\mathbf{u}) d \mathbf{x}^{\prime} d \mathbf{x}=0 .
$$

We note that such results have been established in [22] with the implicit assumption that the integrands are well defined. By the properties of the bilinear form and thus the PD operator, the identity can be stated in the suitable spaces. As $\delta \rightarrow 0$, the problem (2.8) over $\Omega$ converges to Navier-Lamé equation over $\Omega_{s}$ with homogeneous Dirichlet constrained volume condition [24, 40]:

$$
\left\{\begin{aligned}
-\mu \nabla \cdot\left(\nabla \mathbf{u}(\mathbf{x})+\nabla^{T} \mathbf{u}(\mathbf{x})\right)-\mu \nabla(\nabla \cdot \mathbf{u}(\mathbf{x})) & =\mathbf{f}(\mathbf{x}) \quad \text { for } \mathbf{x} \in \Omega_{s} \\
\mathbf{u}(\mathbf{x}) & =0 \quad \text { for } \mathbf{x} \in \partial \Omega_{s}
\end{aligned}\right.
$$


with the coefficient $\mu$, under the above assumption for $\Lambda$, can be evaluated as

$$
\mu=\lim _{\delta \rightarrow 0} \int_{B_{\delta}(0)} \frac{\beta}{|\mathbf{z}|^{2}} z_{1}^{2} z_{2}^{2} d \mathbf{z},
$$

where $\mathbf{z}=\left(z_{1}, \ldots, z_{d}\right)^{T}$.

The weak form of problem (4.6) can be formulated as follows: find $\mathbf{u} \in \mathbf{H}_{0}^{1}(\Omega)\left(=\mathbf{L}_{0}^{2}(\Omega) \cap\left(H^{1}(\Omega)\right)^{d}\right)$ such that for any $\mathbf{v} \in \mathbf{H}_{0}^{1}(\Omega)$ we get

$$
\mu\left(\nabla \mathbf{u}+\nabla^{T} \mathbf{u}: \nabla \mathbf{v}\right)+\mu(\nabla \cdot \mathbf{u}, \nabla \cdot \mathbf{v})=(\mathbf{f}, \mathbf{v}),
$$

where the symbol ':' denotes the Frobenius product.

4.1. A posteriori error analysis. Using a similar finite element discretization as in the last section, we denote $\mathbf{u}^{h} \in\left(\mathbf{S}_{\text {local }}^{h}\right)^{d}$ the finite element approximation of $\mathbf{u}$ by solving (4.8). Applying the framework in [38, we can get a residual-type identity that states, for any $\mathbf{v} \in \mathbf{H}_{0}^{1}(\Omega)$,

$$
\begin{aligned}
\mu((\nabla+ & \left.\left.\nabla^{T}\right)\left(\mathbf{u}-\mathbf{u}^{h}\right): \nabla \mathbf{v}\right)+\mu\left(\nabla \cdot\left(\mathbf{u}-\mathbf{u}^{h}\right), \nabla \cdot \mathbf{v}\right) \\
= & \sum_{K}\left(\int_{K}\left(\mathbf{f}+\mu \nabla \cdot\left(\nabla \mathbf{u}^{h}+\nabla^{T} \mathbf{u}^{h}\right)+\mu \nabla\left(\nabla \cdot \mathbf{u}^{h}\right)\right) \cdot \mathbf{v} d \mathbf{x}\right. \\
& \left.\quad-\frac{1}{2} \int_{\partial K} \mu \llbracket \nabla \mathbf{u}^{h}+\nabla^{T} \mathbf{u}^{h}+\left(\nabla \cdot \mathbf{u}^{h}\right) \mathbf{I} \rrbracket_{\mathbf{r}} \cdot \mathbf{v} d \nu\right),
\end{aligned}
$$

where $\llbracket \nabla \mathbf{u}^{h}+\nabla^{T} \mathbf{u}^{h}+\left(\nabla \cdot \mathbf{u}^{h}\right) \mathbf{I} \rrbracket_{\mathbf{r}}$ denotes the normal flux on an edge (face) $\mathbf{r}$ defined as follows: suppose $\mathbf{r}$ is the common edge (face) of elements $K$ and $K^{\prime}$, then

$$
\begin{aligned}
\llbracket \nabla \mathbf{u}^{h}+\nabla^{T} \mathbf{u}^{h}+\left(\nabla \cdot \mathbf{u}^{h}\right) \mathbf{I} \rrbracket_{\mathbf{r}} & =\left(\nabla \mathbf{u}_{K}^{h}+\nabla^{T} \mathbf{u}_{K}^{h}+\left(\nabla \cdot \mathbf{u}_{K}^{h}\right) \mathbf{I}\right) \cdot \overrightarrow{\mathbf{n}}_{K} \\
& +\left(\nabla \mathbf{u}_{K^{\prime}}^{h}+\nabla^{T} \mathbf{u}_{K^{\prime}}^{h}+\left(\nabla \cdot \mathbf{u}_{K^{\prime}}^{h}\right) \cdot \mathbf{I}\right) \cdot \overrightarrow{\mathbf{n}}_{K^{\prime}} .
\end{aligned}
$$

We now can define the interior residual, similarly as for the scalar case discussed earlier,

$$
\mathbf{r}_{*}^{h}=\mathbf{f}+\mu \nabla \cdot\left(\nabla \mathbf{u}^{h}+\nabla^{T} \mathbf{u}^{h}\right)+\mu \nabla\left(\nabla \cdot \mathbf{u}^{h}\right) \quad \text { in } K,
$$

and the boundary residual

$$
\mathbf{R}_{*}^{h}=\mu \llbracket \nabla \mathbf{u}^{h}+\nabla^{T} \mathbf{u}^{h}+\left(\nabla \cdot \mathbf{u}^{h}\right) \mathbf{I} \rrbracket_{\mathbf{r}} \quad \text { on any interior edge (face) } \mathbf{r},
$$

and then obtain the local a posteriori error estimator correspondingly.

Following the steps in the last section, we get the residual for the peridynamic problem (2.8)

$$
\mathbf{R}^{h}(\mathbf{x})=\mathbf{f}(\mathbf{x})-\mathcal{G}\left(\beta \mathcal{G}^{*}\left(\mathbf{u}^{h}(\mathbf{x})\right)\right), \quad \forall \mathbf{x} \in K,
$$

where $\mathbf{u}^{h} \in\left(\mathbf{S}^{h}\right)^{d}$ is the finite element approximation of $\mathbf{u}$ by solving (4.1). The reliability and efficiency also can be proved as in Section 3.2. We therefore are able to get the following theorem.

Theorem 4.2. Suppose that $\mathbf{u}^{*} \in \mathbf{L}_{n 0}^{2}(\Omega)$ is the weak solution of (4.1), $\mathbf{u}^{h} \in\left(\mathbf{S}^{h}\right)^{d}$ is the finite element approximation of $\mathbf{u}, \mathbf{R}^{h}$ is defined as in (4.13), $\mathbf{e}^{h}=\mathbf{u}^{*}-\mathbf{u}^{h}$ is the exact error. Then there exists nonnegative constants $C_{4}$ and $C_{5}(\delta)$ such that

$$
C_{4} \boldsymbol{\eta}_{\delta}^{h} \leq\left\|e^{h}\right\|_{\mathbf{E}} \leq C_{5}(\delta) \boldsymbol{\eta}_{\delta}^{h},
$$

where $\boldsymbol{\eta}_{\delta}^{h}=\left\|\mathbf{R}^{h}\right\|_{\mathbf{L}^{2}(\Omega)} / \sqrt{M(\delta)}$ denotes the a posteriori error estimator, $\|\cdot\|_{\mathbf{E}}=$ $\sqrt{\mathbf{B}(\cdot, \cdot)}$ denotes the energy norm. 
Next, we will mimic the analysis performed for the nonlocal diffusion problem, and find the connection between (4.13) and (4.9).

4.2. Relation with local case. In this subsection, we use the assumption on $\Omega_{c}$ as in Section 3.3, and further assume that

$$
\begin{array}{r}
\mathbf{u}, \mathbf{v} \in\left(C^{0}(\Omega) \cap H^{1}(\Omega)\right)^{d},\left.\mathbf{u}\right|_{\Omega_{c}}=0,\left.\mathbf{v}\right|_{\Omega_{c}}=0 \\
\mathbf{u}_{K}, \mathbf{v}_{K} \in\left(C^{\tau}(K)\right)^{d} \text { for any } K, \text { where } \tau \geq 2 .
\end{array}
$$

Consider the bilinear form in (4.1). Applying Lemma 4.1 gives

$$
\begin{aligned}
-\int_{\Omega} & \int_{\Omega} \mathcal{G}^{*}(\mathbf{u}) \beta \mathcal{G}^{*}(\mathbf{v}) d \mathbf{x}^{\prime} d \mathbf{x} \\
= & -\int_{\Omega_{s}} \mathbf{v}(\mathbf{x}) \cdot \mathcal{G}\left(\beta \mathcal{G}^{*}(\mathbf{u})\right) d \mathbf{x}^{\prime} d \mathbf{x} \\
= & -\sum_{K} \int_{K} \mathbf{v}(\mathbf{x}) \cdot \mathcal{G}\left(\beta \mathcal{G}^{*}(\mathbf{u})\right) d \mathbf{x}^{\prime} d \mathbf{x} \\
= & \sum_{K} \int_{K} \mathbf{v}(\mathbf{x}) \cdot \int_{K} 2 \Lambda \cdot\left(\mathbf{u}\left(\mathbf{x}^{\prime}\right)-\mathbf{u}(\mathbf{x})\right) d \mathbf{x}^{\prime} d \mathbf{x} \\
& \quad+\sum_{K} \int_{K} \mathbf{v}(\mathbf{x}) \cdot \int_{\Gamma_{K}} 2 \Lambda \cdot\left(\mathbf{u}\left(\mathbf{x}^{\prime}\right)-\mathbf{u}(\mathbf{x})\right) d \mathbf{x}^{\prime} d \mathbf{x} .
\end{aligned}
$$

For any $K$, consider the first term in (4.16),

$$
\begin{aligned}
\int_{K} \mathbf{v}(\mathbf{x}) & \cdot \int_{K} 2 \Lambda \cdot\left(\mathbf{u}\left(\mathbf{x}^{\prime}\right)-\mathbf{u}(\mathbf{x})\right) d \mathbf{x}^{\prime} d \mathbf{x} \\
= & \int_{K} \mathbf{v}(\mathbf{x}) \cdot \int_{K} 2 \Lambda \cdot \nabla \mathbf{u}(\mathbf{x}) \cdot\left(\mathbf{x}^{\prime}-\mathbf{x}\right) d \mathbf{x}^{\prime} d \mathbf{x} \\
& +\int_{K} \mathbf{v}(\mathbf{x}) \cdot \int_{K} 2 \Lambda \cdot \frac{1}{2}\left(\mathbf{x}^{\prime}-\mathbf{x}\right)^{T} H_{\mathbf{u}}(\mathbf{x}) \cdot\left(\mathbf{x}^{\prime}-\mathbf{x}\right) d \mathbf{x}^{\prime} d \mathbf{x} \\
& +\int_{K} \mathbf{v}(\mathbf{x}) \cdot \int_{K} 2 \Lambda \cdot o\left(\delta^{2}\right) d \mathbf{x}^{\prime} d \mathbf{x} \\
= & T_{1}+T_{2}+T_{3},
\end{aligned}
$$

where $H_{\mathbf{u}}(\cdot)$ is the Hessian tensor of $\mathbf{u}$.

Assume $K=K_{\text {in }} \cup K_{\text {out }}$, where $K_{\text {in }}$ and $K_{\text {out }}$ are defined as before. Denote $\mathbf{z}=\mathbf{x}^{\prime}-\mathbf{x}$, and evaluate $T_{2}$ as

$$
\begin{aligned}
& \int_{K} \mathbf{v}(\mathbf{x}) \cdot \int_{K} 2 \Lambda \cdot \frac{1}{2}\left(\mathbf{x}^{\prime}-\mathbf{x}\right)^{T} H_{\mathbf{u}}(\mathbf{x})\left(\mathbf{x}^{\prime}-\mathbf{x}\right) d \mathbf{x}^{\prime} d \mathbf{x} \\
& \quad=\int_{K_{i n}} \mathbf{v}(\mathbf{x}) \cdot \int_{B_{\delta}(0)} \frac{\beta}{|\mathbf{z}|^{2}} \mathbf{z} \otimes \mathbf{z} \cdot \mathbf{z}^{T} H_{\mathbf{u}}(\mathbf{x}) \mathbf{z} d \mathbf{z} d \mathbf{x}+o(1) .
\end{aligned}
$$

Let $\mathbf{z}=\left(z_{1}, z_{2}, \ldots, z_{d}\right)^{T}, \mathbf{u}=\left(u_{1}, u_{2}, \ldots, u_{d}\right)^{T}$, then we can compute the $i$ th component of $\left(\mathbf{z} \otimes \mathbf{z} \cdot \mathbf{z}^{T} H_{\mathbf{u}}(\mathbf{x}) \mathbf{z}\right)$ as

$$
\left(\mathbf{z} \otimes \mathbf{z} \cdot \mathbf{z}^{T} H_{\mathbf{u}}(\mathbf{x}) \mathbf{z}\right)_{i}=\sum_{j, k, l} z_{i} z_{j} z_{k} z_{l} u_{l, j k}(\mathbf{x}),
$$

where $u_{l, j k}$ denotes the second-order mixed derivative of $u_{l}$ along $x_{j}$ and $x_{k}$ directions. 
Using the symmetry of integral over $B_{\delta}(0)$, the only non-zero terms from (4.19) after integration, are

$$
\begin{aligned}
\int_{B_{\delta}(0)} & \frac{\beta}{|\mathbf{z}|^{2}}\left(z_{i}^{4} u_{i, i i}(\mathbf{x})+\sum_{j \neq i} z_{i}^{2} z_{j}^{2}\left(u_{i, j j}(\mathbf{x})+u_{j, i j}(\mathbf{x})+u_{j, j i}(\mathbf{x})\right)\right) d \mathbf{z} \\
= & \left(\int_{B_{\delta}(0)} \frac{\beta}{|\mathbf{z}|^{2}} z_{1}^{2} z_{2}^{2} d \mathbf{z}\right)\left(3 u_{i, i i}(\mathbf{x})+\sum_{j \neq i}\left(u_{i, j j}(\mathbf{x})+u_{j, i j}(\mathbf{x})+u_{j, j i}(\mathbf{x})\right)\right) \\
= & \left(\int_{B_{\delta}(0)} \frac{\beta}{|\mathbf{z}|^{2}} z_{1}^{2} z_{2}^{2} d \mathbf{z}\right) \sum_{j}\left(u_{i, j j}(\mathbf{x})+u_{j, i j}(\mathbf{x})+u_{j, j i}(\mathbf{x})\right) \\
= & \left(\int_{B_{\delta}(0)} \frac{\beta}{|\mathbf{z}|^{2}} z_{1}^{2} z_{2}^{2} d \mathbf{z}\right)\left(\nabla \cdot\left(\nabla \mathbf{u}+\nabla^{T} \mathbf{u}\right)+\nabla(\nabla \cdot \mathbf{u})\right)_{i},
\end{aligned}
$$

since we have the relationship

$$
\int_{B_{\delta}(0)} \beta \frac{z_{l}^{4}}{|\mathbf{z}|^{2}} d \mathbf{z}=\int_{B_{\delta}(0)} \beta \frac{z_{s}^{4}}{|\mathbf{z}|^{2}} d \mathbf{z}=3 \int_{B_{\delta}(0)} \beta \frac{z_{m}^{2} z_{n}^{2}}{|\mathbf{z}|^{2}} d \mathbf{z}=3 \int_{B_{\delta}(0)} \beta \frac{z_{t}^{2} z_{p}^{2}}{|\mathbf{z}|^{2}} d \mathbf{z},
$$

for any $l, m, n(m \neq n), s, t, p(t \neq p)=1,2, \ldots, d$.

Therefore, as $\delta \rightarrow 0$,

$$
T_{2} \rightarrow \int_{K_{\text {in }}} \mathbf{v}(\mathbf{x}) \cdot \mu\left(\nabla \cdot\left(\nabla \mathbf{u}+\nabla^{T} \mathbf{u}\right)+\nabla(\nabla \cdot \mathbf{u})\right) d \mathbf{x} .
$$

For the second term in (4.16), we have

$$
\begin{aligned}
\int_{K} \mathbf{v}(\mathbf{x}) \cdot \int_{\Gamma_{K}} 2 \Lambda \cdot\left(\mathbf{u}\left(\mathbf{x}^{\prime}\right)-\mathbf{u}(\mathbf{x})\right) d \mathbf{x}^{\prime} d \mathbf{x} \\
=\int_{K} \mathbf{v}(\mathbf{x}) \cdot \int_{\Gamma_{K}} 2 \Lambda \cdot \nabla \mathbf{u}_{K}(\mathbf{x}) \cdot\left(\mathbf{x}^{\prime}-\mathbf{x}\right) d \mathbf{x}^{\prime} d \mathbf{x} \\
\quad+\int_{K} \mathbf{v}(\mathbf{x}) \cdot \int_{\Gamma_{K}} 2 \Lambda \cdot\left[\nabla \mathbf{u}_{\Gamma_{K}}(\mathbf{s})-\nabla \mathbf{u}_{K}(\mathbf{x})\right] \cdot\left(\mathbf{x}^{\prime}-\mathbf{s}\right) d \mathbf{x}^{\prime} d \mathbf{x} \\
\quad+\int_{K} \mathbf{v}(\mathbf{x}) \cdot \int_{\Gamma_{K}} 2 \Lambda \cdot o(\delta) d \mathbf{x}^{\prime} d \mathbf{x} \\
=S_{1}+S_{2}+S_{3} .
\end{aligned}
$$

Adding up $T_{1}$ and $S_{1}$ gives us

$$
\begin{aligned}
T_{1}+S_{1} & =\int_{K} \mathbf{v}(\mathbf{x}) \cdot \int_{K \cup \Gamma_{K}} 2 \Lambda \cdot \nabla \mathbf{u}_{K}(\mathbf{x}) \cdot\left(\mathbf{x}^{\prime}-\mathbf{x}\right) d \mathbf{x}^{\prime} d \mathbf{x} \\
& =\int_{K} \mathbf{v}(\mathbf{x}) \cdot \int_{B_{\delta}(0)} 2 \Lambda \cdot \nabla \mathbf{u}(\mathbf{x}) \cdot \mathbf{z} d \mathbf{z} d \mathbf{x}, \\
& =0 .
\end{aligned}
$$

By the symmetry of $B_{\delta}(0)$, it is also not hard to show (considering the scaling factor in $\beta$ ) that

$$
T_{3}+S_{3} \rightarrow 0 \quad \text { as } \quad \delta \rightarrow 0 .
$$


Next, we will evaluate the term $S_{2}$ by partly mimicking the argument made in Section 3.3 Assume that the elements $K$ and $K^{\prime}$ have a common edge e and the outward normal of $K$ on $\mathbf{e}$ is $\overrightarrow{\mathbf{n}}_{\mathbf{e}}=(1,0,0, \ldots, 0)^{T}$. Since $\mathbf{v}(\cdot)$ is continuous across elements, we then have

$$
\begin{aligned}
S_{2}= & \int_{K} \mathbf{v}(\mathbf{x}) \cdot \int_{\Gamma_{K}} 2 \Lambda \cdot\left(\nabla \mathbf{u}_{\Gamma_{K}}(\mathbf{s})-\nabla \mathbf{u}_{K}(\mathbf{x})\right) \cdot\left(\mathbf{x}^{\prime}-\mathbf{s}\right) d \mathbf{x}^{\prime} d \mathbf{x} \\
= & \sum_{\mathbf{r} \in \text { edge }(K) /\{\mathbf{e}\}} \int_{K_{\text {out }} \cap B_{\delta}(\mathbf{r})} \mathbf{v}(\mathbf{x}) \cdot \int_{\Gamma_{K} \cap B_{\delta}(\mathbf{x})} 2 \Lambda \cdot \\
& +\int_{\mathbf{e}} \mathbf{v}\left(\mathbf{x}_{\mathbf{e}}\right) \cdot \int_{-\delta}^{0} \int_{B_{\delta}(\mathbf{x}) \cap \Gamma_{K}} 2 \Lambda \cdot \\
& \left(\nabla \mathbf{u}_{\Gamma_{K}}(\mathbf{s})-\nabla \mathbf{u}_{K}(\mathbf{x})\right) \cdot\left(\mathbf{x}^{\prime}-\mathbf{s}\right) d \mathbf{x}^{\prime} d \mathbf{x} \\
& \left(\nabla \mathbf{u}_{\Gamma_{K}}\left(\mathbf{x}_{\mathbf{e}}\right)-\nabla \mathbf{u}_{K}\left(\mathbf{x}_{\mathbf{e}}\right)\right) \cdot\left(\mathbf{x}^{\prime}-\mathbf{s}\right) d \mathbf{x}^{\prime} d \xi d \vec{\eta}+o(1),
\end{aligned}
$$

where $\mathbf{x}_{\mathbf{e}}=(\xi, 0)$. For the $K^{\prime}$ part, we change the order of integration and obtain

$$
\begin{aligned}
S_{2}^{\prime}= & \int_{K^{\prime}} \mathbf{v}(\mathbf{x}) \cdot \int_{\Gamma_{K^{\prime}}} 2 \Lambda \cdot\left(\nabla \mathbf{u}_{\Gamma_{K^{\prime}}}(\mathbf{s})-\nabla \mathbf{u}_{K^{\prime}}(\mathbf{x})\right) \cdot\left(\mathbf{x}^{\prime}-\mathbf{s}\right) d \mathbf{x}^{\prime} d \mathbf{x} \\
= & \sum_{\mathbf{r} \in \text { edge }\left(K^{\prime}\right) /\{\mathbf{e}\}} \int_{K_{\text {out }}^{\prime} \cap B_{\delta}(\mathbf{r})} \mathbf{v}(\mathbf{x}) \cdot \int_{\Gamma_{K^{\prime}} \cap B_{\delta}(\mathbf{x})} 2 \Lambda \cdot \\
& +\int_{\mathbf{e}} \mathbf{v}\left(\mathbf{x}_{\mathbf{e}}\right) \cdot \int_{-\delta}^{0} \int_{B_{\delta}(\mathbf{x}) \cap \Gamma_{K}} 2 \Lambda \cdot \\
& \left.\left(\nabla \mathbf{u}_{\Gamma_{K^{\prime}}}(\mathbf{s})-\nabla \mathbf{u}_{K^{\prime}}(\mathbf{x})\right) \cdot\left(\mathbf{x}_{\mathbf{e}}\right)-\mathbf{s}\right) d \mathbf{x}^{\prime} d \mathbf{x}
\end{aligned}
$$

Adding up (4.25) and (4.26), the resulting integral on edge (face) e can be presented as

$$
\int_{\mathbf{e}} \mathbf{v}\left(\mathbf{x}_{\mathbf{e}}\right) \cdot \int_{-\delta}^{0} \int_{S C(\delta, \delta+\xi)} \frac{2 \beta}{|\mathbf{z}|^{2}} \mathbf{z} \otimes \mathbf{z} \nabla \tilde{\mathbf{u}}\left(\mathbf{x}_{\mathbf{e}}\right) \mathbf{z} d \mathbf{z} d \xi d \vec{\eta}+o(1),
$$

where $\mathbf{z}=\mathbf{x}^{\prime}-\mathbf{x}, \nabla \tilde{\mathbf{u}}(\cdot)=\nabla \mathbf{u}_{\Gamma_{K}}(\cdot)-\nabla \mathbf{u}_{K}(\cdot)$. Notice the contributions from $S_{2}$ and $S_{2}^{\prime}$ to (4.27) are the same as $\delta \rightarrow 0$.

The $i$ th component of $\mathbf{z} \otimes \mathbf{z} \nabla \tilde{\mathbf{u}}\left(\mathbf{x}_{\mathbf{e}}\right) \mathbf{z}$ can be computed as

$$
\left(\mathbf{z} \otimes \mathbf{z} \nabla \tilde{\mathbf{u}}\left(\mathbf{x}_{\mathbf{e}}\right) \mathbf{z}\right)_{i}=\sum_{j, k=1}^{d} z_{i} z_{j} z_{k} \tilde{u}_{j, k}\left(\mathbf{x}_{\mathbf{e}}\right),
$$

where $\tilde{u}_{j, k}$ denotes the derivative of $\left.u_{j}\right|_{\Gamma_{K}}-\left.u_{j}\right|_{K}$ along the $x_{k}$ direction. Since the outward normal of $K$ on $\mathbf{e}$ is $(1,0,0, \ldots, 0)^{T}$, by the symmetry of the integral, when $i=1$ we have

$$
\begin{aligned}
& \int_{-\delta}^{0} \int_{B_{\delta}(\mathbf{x}) \cap \Gamma_{K}} \frac{2 \beta}{|\mathbf{z}|^{2}}\left(\sum_{j, k=1}^{d} z_{i} z_{j} z_{k} \tilde{u}_{j, k}\left(\mathbf{x}_{\mathbf{e}}\right)\right) d \mathbf{z} d \eta \\
& \quad=\int_{-\delta}^{0} \int_{B_{\delta}(\mathbf{x}) \cap \Gamma_{K}} \frac{2 \beta}{|\mathbf{z}|^{2}}\left(\sum_{j=1}^{d} z_{1} z_{j}^{2} \tilde{u}_{j, j}\left(\mathbf{x}_{\mathbf{e}}\right)\right) d \mathbf{z} d \eta
\end{aligned}
$$




$$
\begin{aligned}
& =\int_{B_{\delta}\left(\mathbf{x}_{\mathbf{e}}\right) \cap \Gamma_{K}} \int_{-z_{1}}^{0} \frac{2 \beta}{|\mathbf{z}|^{2}}\left(\sum_{j=1}^{d} z_{1} z_{j}^{2} \tilde{u}_{j, j}\left(\mathbf{x}_{\mathbf{e}}\right)\right) d \eta d \mathbf{z} \\
& =\int_{B_{\delta}(0)} \frac{\beta}{|\mathbf{z}|^{2}}\left(\sum_{j=1}^{d} z_{1}^{2} z_{j}^{2} \tilde{u}_{j, j}\left(\mathbf{x}_{\mathbf{e}}\right)\right) d \mathbf{z} \\
& =\left(\int_{B_{\delta}(0)} \frac{\beta}{|\mathbf{z}|^{2}} z_{1}^{2} z_{2}^{2} d \mathbf{z}\right)\left(3 \tilde{u}_{1,1}\left(\mathbf{x}_{\mathbf{e}}\right)+\sum_{j=2}^{d} \tilde{u}_{j, j}\left(\mathbf{x}_{\mathbf{e}}\right)\right) .
\end{aligned}
$$

Similarly, when $i>1$ we get

$$
\begin{aligned}
\int_{-\delta}^{0} & \int_{B_{\delta}(\mathbf{x}) \cap \Gamma_{K}} \frac{2 \beta}{|\mathbf{z}|^{2}}\left(\sum_{j, k=1}^{d} z_{i} z_{j} z_{k} \tilde{u}_{j, k}\left(\mathbf{x}_{\mathbf{e}}\right)\right) d \mathbf{z} d \eta \\
& =\int_{-\delta}^{0} \int_{B_{\delta}(\mathbf{x}) \cap \Gamma_{K}} \frac{2 \beta}{|\mathbf{z}|^{2}}\left(z_{1} z_{i}^{2}\left(\tilde{u}_{1, i}\left(\mathbf{x}_{\mathbf{e}}\right)+\tilde{u}_{i, 1}\left(\mathbf{x}_{\mathbf{e}}\right)\right) d \mathbf{z} d \eta\right. \\
& =\int_{B_{\delta}(0)} \frac{\beta}{|\mathbf{z}|^{2}}\left(z_{1}^{2} z_{i}^{2}\left(\tilde{u}_{1, i}\left(\mathbf{x}_{\mathbf{e}}\right)+\tilde{u}_{i, 1}\left(\mathbf{x}_{\mathbf{e}}\right)\right) d \mathbf{z}\right. \\
& =\left(\int_{B_{\delta}(0)} \frac{\beta}{|\mathbf{z}|^{2}} z_{1}^{2} z_{2}^{2} d \mathbf{z}\right)\left(\tilde{u}_{1, i}\left(\mathbf{x}_{\mathbf{e}}\right)+\tilde{u}_{i, 1}\left(\mathbf{x}_{\mathbf{e}}\right)\right)
\end{aligned}
$$

Combining (4.29) and (4.30), we get that the expression in (4.27) becomes

$$
\int_{\mathbf{e}} \mathbf{v}\left(\mathbf{x}_{\mathbf{e}}\right) \cdot \mu_{\delta}\left(\nabla \tilde{\mathbf{u}}\left(\mathbf{x}_{\mathbf{e}}\right)+\nabla \tilde{\mathbf{u}}^{T}\left(\mathbf{x}_{\mathbf{e}}\right)+\left(\nabla \cdot \tilde{\mathbf{u}}\left(\mathbf{x}_{\mathbf{e}}\right)\right) \mathbf{I}\right) \cdot \overrightarrow{\mathbf{n}}_{\mathbf{e}} d \xi+o(1)
$$

where

$$
\mu_{\delta}=\int_{B_{\delta}(0)} \frac{\beta}{|\mathbf{z}|^{2}} z_{1}^{2} z_{2}^{2} d \mathbf{z}
$$

So

$$
S_{2} \quad \rightarrow \quad-\frac{1}{2} \int_{\partial K} \mathbf{v}(\nu) \cdot \mu \llbracket \nabla \mathbf{u}(\nu)+\nabla^{T} \mathbf{u}(\nu)+(\nabla \cdot \mathbf{u}(\nu)) \mathbf{I} \rrbracket_{\mathbf{r}} d \nu
$$

where $\mu=\lim _{\delta \rightarrow 0} \mu_{\delta}$.

Finally, combining (4.16), (4.17), (4.21), (4.23) and (4.31), we have

Theorem 4.3. Under the assumption (4.3), with $\mathbf{u}$ and $\mathbf{v}$ satisfying (4.15). For any element $K$, as $\delta \rightarrow 0$,

$$
\begin{gathered}
-\int_{K} \mathbf{v}(\mathbf{x}) \cdot \mathcal{G}\left(\beta \mathcal{G}^{*}(\mathbf{u})\right) d \mathbf{x}^{\prime} d \mathbf{x} \\
\rightarrow \quad \int_{K} \mathbf{v}(\mathbf{x}) \cdot\left(\mu\left(\nabla \cdot\left(\nabla \mathbf{u}+\nabla^{T} \mathbf{u}\right)+\nabla(\nabla \cdot \mathbf{u})\right)\right) d \mathbf{x} \\
\quad-\frac{1}{2} \int_{\partial K} \mathbf{v}(\nu) \cdot \mu \llbracket \nabla \mathbf{u}(\nu)+\nabla^{T} \mathbf{u}(\nu)+(\nabla \cdot \mathbf{u}(\nu)) \mathbf{I} \rrbracket_{\mathbf{r}} d \nu
\end{gathered}
$$


We therefore have the corollary

Corollary 4.4. Under assumption (4.3), with $\mathbf{u}^{h}$ and $\mathbf{v}$ satisfy (4.15). For any element $K$, as $\delta \rightarrow 0$,

$$
\int_{K} \mathbf{v}(\mathbf{x}) \cdot \mathbf{R}^{h} d \mathbf{x}^{\prime} d \mathbf{x} \rightarrow \int_{K} \mathbf{v}(\mathbf{x}) \cdot \mathbf{r}_{*}^{h} d \mathbf{x}-\frac{1}{2} \int_{\partial K} \mathbf{v}(\nu) \cdot \mathbf{R}_{*}^{h} d \nu .
$$

Thus we can see that the a posteriori error estimation for the PD bond-based model is connected to its local counterpart as well. Summing up (4.33) for all the $K$ 's implies the local limit of PD operator being the Navier operator (with Poisson ratio $1 / 4)$, as stated in [22].

\section{NumericAl EXPERIMENTS}

In this section, we compute the finite element approximations and the a posteriori error approximations for several nonlocal diffusion equations to validate our theoretical analysis. Notice that the numerical experiments are performed in 1D and 2D, while our theoretical analysis is true for any dimension. To numerically evaluate the residual term as defined in (3.13) and its $L^{2}$ norm, one needs to use numerical quadratures for the integral operator, details can be found in $[20$.

\subsection{One-dimensional numerical experiments.}

Example 5.1. We consider the following 1D problem by choosing $\boldsymbol{\alpha}=\frac{x^{\prime}-x}{\left|x^{\prime}-x\right|}$ and $\beta=-\frac{1}{\delta^{3}}$,

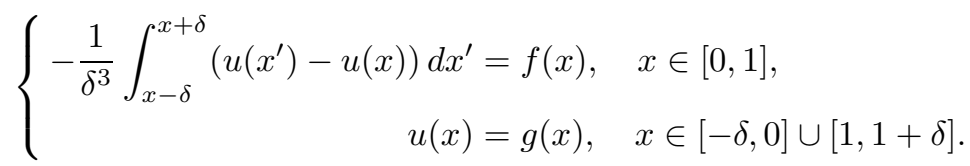

We choose the exact solution to be

$$
u(x)=-x^{3}+x^{2},
$$

therefore

$$
f(x)=2 x-\frac{2}{3} .
$$

Also, we set $\delta=2,0.2,0.02$, respectively, and restriction condition $g(x)$ can be determined by $u(x)$ and $\delta$ accordingly. Then we can compute $M(\delta)$ as defined in (2.9) by

$$
M(\delta)=\int_{-\delta}^{\delta} \frac{1}{\delta^{3}} d z=\frac{2}{\delta^{2}},
$$

so $M(\delta)=0.5,50,5000$ for different $\delta$.

We use the continuous Galerkin method to solve problem (5.1) with continuous piecewise linear basis functions, one can refer to 20] for more implementation details. For each $\delta$, we divide the interval $[0,1]$ into $N$ elements, where $N=25,50,100,200,400$, respectively. The numerical results can be found in Table 1, where we list the error in $L^{2}$ norm, in energy norm, and a posteriori error estimator. The convergence rates (CR) for each error are computed as well as the ratio between $\eta_{\delta}^{h}$ and $\left\|e^{h}\right\|_{E}$ to examine reliability and efficiency of the estimator. We can observe that our a posteriori error estimator approximates $\left\|e^{h}\right\|_{E}$ robustly, the ratio between the two is very stable for each $\delta$, so our estimator is both reliable 
TABLE 1. Numerical results with different $\delta$ in Example 5.1

\begin{tabular}{|c|c|c|c|c|c|c|c|c|}
\hline \multicolumn{9}{|c|}{$\delta=2$} \\
\hline $\mathrm{N}$ & $\left\|e^{h}\right\|_{L^{2}}$ & $\mathrm{CR}$ & $\left\|e^{h}\right\|_{E}$ & $\mathrm{CR}$ & $\eta_{\delta}^{h}$ & $\mathrm{CR}$ & $\eta_{\delta}^{h} /\left\|e^{h}\right\|_{L^{2}}$ & $\eta_{\delta}^{h} /\left\|e_{h}\right\|_{E}$ \\
\hline 25 & $2.92 \mathrm{e}-04$ & - & $8.45 \mathrm{e}-05$ & - & $8.09 \mathrm{e}-05$ & - & 0.28 & 0.96 \\
\hline 50 & $7.30 \mathrm{e}-05$ & 2.00 & $2.11 \mathrm{e}-05$ & 2.00 & $2.02 \mathrm{e}-05$ & 2.00 & 0.28 & 0.96 \\
\hline 100 & $1.83 \mathrm{e}-05$ & 2.00 & $5.28 \mathrm{e}-06$ & 2.00 & $5.05 \mathrm{e}-06$ & 2.00 & 0.28 & 0.96 \\
\hline 200 & $4.56 \mathrm{e}-06$ & 2.00 & $1.32 \mathrm{e}-06$ & 2.00 & $1.26 \mathrm{e}-06$ & 2.00 & 0.28 & 0.96 \\
\hline 400 & $1.14 \mathrm{e}-06$ & 2.00 & $3.30 \mathrm{e}-07$ & 2.00 & $3.16 \mathrm{e}-07$ & 2.00 & 0.28 & 0.96 \\
\hline \multicolumn{9}{|c|}{$\delta=0.2$} \\
\hline $\mathrm{N}$ & $\left\|e^{h}\right\|_{L^{2}}$ & CR & $\left\|e^{h}\right\|_{E}$ & $\mathrm{CR}$ & $\eta_{\delta}^{h}$ & $\mathrm{CR}$ & $\eta_{\delta}^{h} /\left\|e^{h}\right\|_{L^{2}}$ & $\eta_{\delta}^{h} /\left\|e^{h}\right\|_{E}$ \\
\hline 25 & $2.92 \mathrm{e}-04$ & - & $8.45 \mathrm{e}-04$ & - & $8.09 \mathrm{e}-04$ & - & 2.77 & 0.96 \\
\hline 50 & $7.30 \mathrm{e}-05$ & 2.00 & $2.11 \mathrm{e}-04$ & 2.00 & $2.02 \mathrm{e}-04$ & 2.00 & 2.77 & 0.96 \\
\hline 100 & $1.83 \mathrm{e}-05$ & 2.00 & $5.28 \mathrm{e}-05$ & 2.00 & $5.05 e-05$ & 2.00 & 2.77 & 0.96 \\
\hline 200 & $4.56 \mathrm{e}-06$ & 2.00 & $1.32 \mathrm{e}-05$ & 2.00 & $1.26 \mathrm{e}-05$ & 2.00 & 2.77 & 0.96 \\
\hline 400 & $1.14 \mathrm{e}-06$ & 2.00 & $3.30 \mathrm{e}-06$ & 2.00 & $3.16 \mathrm{e}-06$ & 2.00 & 2.77 & 0.96 \\
\hline \multicolumn{9}{|c|}{$\delta=0.02$} \\
\hline $\mathrm{N}$ & $\left\|e^{h}\right\|_{L^{2}}$ & $\mathrm{CR}$ & $\left\|e^{h}\right\|_{E}$ & $\mathrm{CR}$ & $\eta_{\delta}^{h}$ & $\mathrm{CR}$ & $\eta_{\delta}^{h} /\left\|e^{h}\right\|_{L^{2}}$ & $\eta_{\delta}^{h} /\left\|e^{h}\right\|_{E}$ \\
\hline 25 & $2.92 \mathrm{e}-04$ & - & $8.45 \mathrm{e}-03$ & - & $8.09 \mathrm{e}-03$ & - & 27.68 & 0.96 \\
\hline 50 & $7.30 \mathrm{e}-05$ & 2.00 & $2.11 \mathrm{e}-03$ & 2.00 & $2.02 \mathrm{e}-03$ & 2.00 & 27.68 & 0.96 \\
\hline 100 & $1.83 \mathrm{e}-05$ & 2.00 & $5.28 \mathrm{e}-04$ & 2.00 & $5.05 \mathrm{e}-04$ & 2.00 & 27.68 & 0.96 \\
\hline 200 & $4.56 \mathrm{e}-06$ & 2.00 & $1.32 \mathrm{e}-04$ & 2.00 & $1.26 \mathrm{e}-04$ & 2.00 & 27.68 & 0.96 \\
\hline 400 & $1.14 \mathrm{e}-06$ & 2.00 & $3.30 \mathrm{e}-05$ & 2.00 & $3.16 \mathrm{e}-05$ & 2.00 & 27.68 & 0.96 \\
\hline
\end{tabular}

and efficient, it also verifies the conclusion we discussed in Remark 3.10. This numerical experiment is consistent with Theorem 3.4. One can also observe that the ratio between $\eta_{\delta}^{h}$ and $\left\|e^{h}\right\|_{L^{2}}$ is also stable for fixed $\delta$, while the ratio is proportional to $\sqrt{M(\delta)}$, which is not surprising given the loss of equivalence between the energy norm and the $L^{2}$ norm in the local limit.

Example 5.2. We use the same problem as in Example 5.1 but with a discontinuous exact solution

$$
u(x)=\left\{\begin{array}{cc}
x, & x<0.5, \\
x^{2}, & \text { otherwise } .
\end{array}\right.
$$

Therefore, $f(x)$ can be found as

$$
f(x)= \begin{cases}0, & x<0.5-\delta, \\ -\frac{1}{\delta^{3}}\left(\frac{(x+\delta)^{3}}{3}-\frac{(x+\delta)^{2}}{2}+\frac{1}{12}\right), & 0.5-\delta \leq x<0.5, \\ -\frac{1}{\delta^{3}}\left(\frac{(x-\delta)^{3}}{3}-\frac{(x-\delta)^{2}}{2}+\frac{1}{12}\right)-\frac{2}{3}, & 0.5 \leq x<0.5+\delta, \\ -\frac{2}{3}, & \text { otherwise. }\end{cases}
$$

We study the numerical solution with continuous piecewise linear basis functions and $\delta=2,0.2,0.02$. 
TABLE 2. Numerical results with different $\delta$ in Example 5.2

\begin{tabular}{|c|c|c|c|c|c|c|c|c|}
\hline \multicolumn{9}{|c|}{$\delta=2$} \\
\hline $\mathrm{N}$ & $\left\|e^{h}\right\|_{L^{2}}$ & $\mathrm{CR}$ & $\left\|e^{h}\right\|_{E}$ & $\mathrm{CR}$ & $\eta_{\delta}^{h}$ & $\mathrm{CR}$ & $\eta_{\delta}^{h} /\left\|e^{h}\right\|_{L^{2}}$ & $\eta_{\delta}^{h} /\left\|e_{h}\right\|_{E}$ \\
\hline 25 & $1.38 \mathrm{e}-02$ & - & $9.77 \mathrm{e}-03$ & - & $9.77 \mathrm{e}-03$ & - & 0.71 & 1.00 \\
\hline 50 & $1.34 \mathrm{e}-02$ & 0.04 & $9.34 \mathrm{e}-03$ & 0.07 & $9.34 \mathrm{e}-03$ & 0.07 & 0.69 & 1.00 \\
\hline 100 & $9.50 \mathrm{e}-03$ & 0.50 & $6.60 \mathrm{e}-03$ & 0.50 & $6.60 \mathrm{e}-03$ & 0.50 & 0.69 & 1.00 \\
\hline 200 & $6.72 \mathrm{e}-03$ & 0.50 & $4.67 \mathrm{e}-03$ & 0.50 & $4.67 \mathrm{e}-03$ & 0.50 & 0.69 & 1.00 \\
\hline 400 & $4.75 \mathrm{e}-03$ & 0.50 & $3.30 \mathrm{e}-03$ & 0.50 & $3.30 \mathrm{e}-03$ & 0.50 & 0.69 & 1.00 \\
\hline \multicolumn{9}{|c|}{$\delta=0.2$} \\
\hline $\mathrm{N}$ & $\left\|e^{h}\right\|_{L^{2}}$ & $\mathrm{CR}$ & $\left\|e^{h}\right\|_{E}$ & $\overline{C R}$ & $\eta_{\delta}^{h}$ & $\mathrm{CR}$ & $\overline{\eta_{\delta}^{h} /\left\|e^{h}\right\|_{L^{2}}}$ & $\eta_{\delta}^{h} /\left\|e^{h}\right\|_{E}$ \\
\hline 25 & $1.97 \mathrm{e}-02$ & - & $1.01 \mathrm{e}-01$ & - & $9.78 \mathrm{e}-02$ & - & 4.97 & 0.97 \\
\hline 50 & $1.34 \mathrm{e}-02$ & 0.55 & $9.34 \mathrm{e}-02$ & 0.11 & $9.33 \mathrm{e}-02$ & 0.07 & 6.95 & 1.00 \\
\hline 100 & $9.50 \mathrm{e}-03$ & 0.50 & $6.60 \mathrm{e}-02$ & 0.50 & $6.60 \mathrm{e}-02$ & 0.50 & 6.95 & 1.00 \\
\hline 200 & $6.72 \mathrm{e}-03$ & 0.50 & $4.67 \mathrm{e}-02$ & 0.50 & $4.67 \mathrm{e}-02$ & 0.50 & 6.95 & 1.00 \\
\hline 400 & $4.75 \mathrm{e}-03$ & 0.50 & $3.30 \mathrm{e}-02$ & 0.50 & $3.30 \mathrm{e}-02$ & 0.50 & 6.95 & 1.00 \\
\hline \multicolumn{9}{|c|}{$\delta=0.02$} \\
\hline $\mathrm{N}$ & $\left\|e^{h}\right\|_{L^{2}}$ & $\mathrm{CR}$ & $\left\|e^{h}\right\|_{E}$ & $\mathrm{CR}$ & $\eta_{\delta}^{h}$ & $\mathrm{CR}$ & $\eta_{\delta}^{h} /\left\|e^{h}\right\|_{L^{2}}$ & $\eta_{\delta}^{h} /\left\|e^{h}\right\|_{E}$ \\
\hline 25 & $1.94 \mathrm{e}-02$ & - & $1.36 \mathrm{e}+00$ & - & $1.32 \mathrm{e}+00$ & - & 67.92 & 0.97 \\
\hline 50 & $1.36 \mathrm{e}-02$ & 0.52 & $9.61 \mathrm{e}-01$ & 0.50 & $9.89 \mathrm{e}-01$ & 0.42 & 72.84 & 1.03 \\
\hline 100 & $9.51 \mathrm{e}-03$ & 0.51 & $6.53 \mathrm{e}-01$ & 0.56 & $6.47 \mathrm{e}-01$ & 0.61 & 67.97 & 0.99 \\
\hline 200 & $6.72 \mathrm{e}-03$ & 0.50 & $4.66 \mathrm{e}-01$ & 0.49 & $4.66 \mathrm{e}-01$ & 0.47 & 69.31 & 1.00 \\
\hline 400 & $4.75 \mathrm{e}-03$ & 0.50 & $3.30 \mathrm{e}-01$ & 0.50 & $3.30 \mathrm{e}-01$ & 0.50 & 69.46 & 1.00 \\
\hline
\end{tabular}

The interval $[0,1]$ is divided into $N=25,50,100,200,400$ subintervals, respectively, and numerical results can be found in Table2. The convergence rate of $\left\|e^{h}\right\|_{E}$ is found to be about $1 / 2$ (consistent with the numerical experiments in 20]). The a posteriori error estimator has the same convergence rate, and the ratio between the two remains almost a constant along with the increase of $N$, which indicates the reliability and efficiency numerically. It again reveals that the ratios $\eta_{\delta}^{h} /\left\|e^{h}\right\|_{L^{2}}$ are proportional to $\delta^{-1}$, similar to the previous examples.

Since the exact solution is discontinuous, our error estimator should serve to capture the discontinuity (at 0.5). Figures 6 and 7 show distributions of $\log _{10}\left(\left\|e^{h}\right\|_{E}\right)$ and $\log _{10}\left(\eta^{h}\right)$ for all the grids over $[0,1]$, with different $\delta$ and $N$. It can be observed that our error estimator approximates the exact error very well, and the larger $N$ is, the better the estimator becomes in capturing the discontinuity.

Example 5.3. In this example, we resolve Example 5.2 with discontinuous piecewise linear basis functions and $\delta=2,0.2,0.02$. Computational results are listed in Table 3, As discussed in [20], we get second order convergence for $L^{2}$ norm of error with discontinuous piecewise linear basis, and we also get second order convergence for both energy norm of error and posteriori error estimator. Notice that, for Examples 5.2 and 5.3 the discontinuity point is on one of the grid points. 

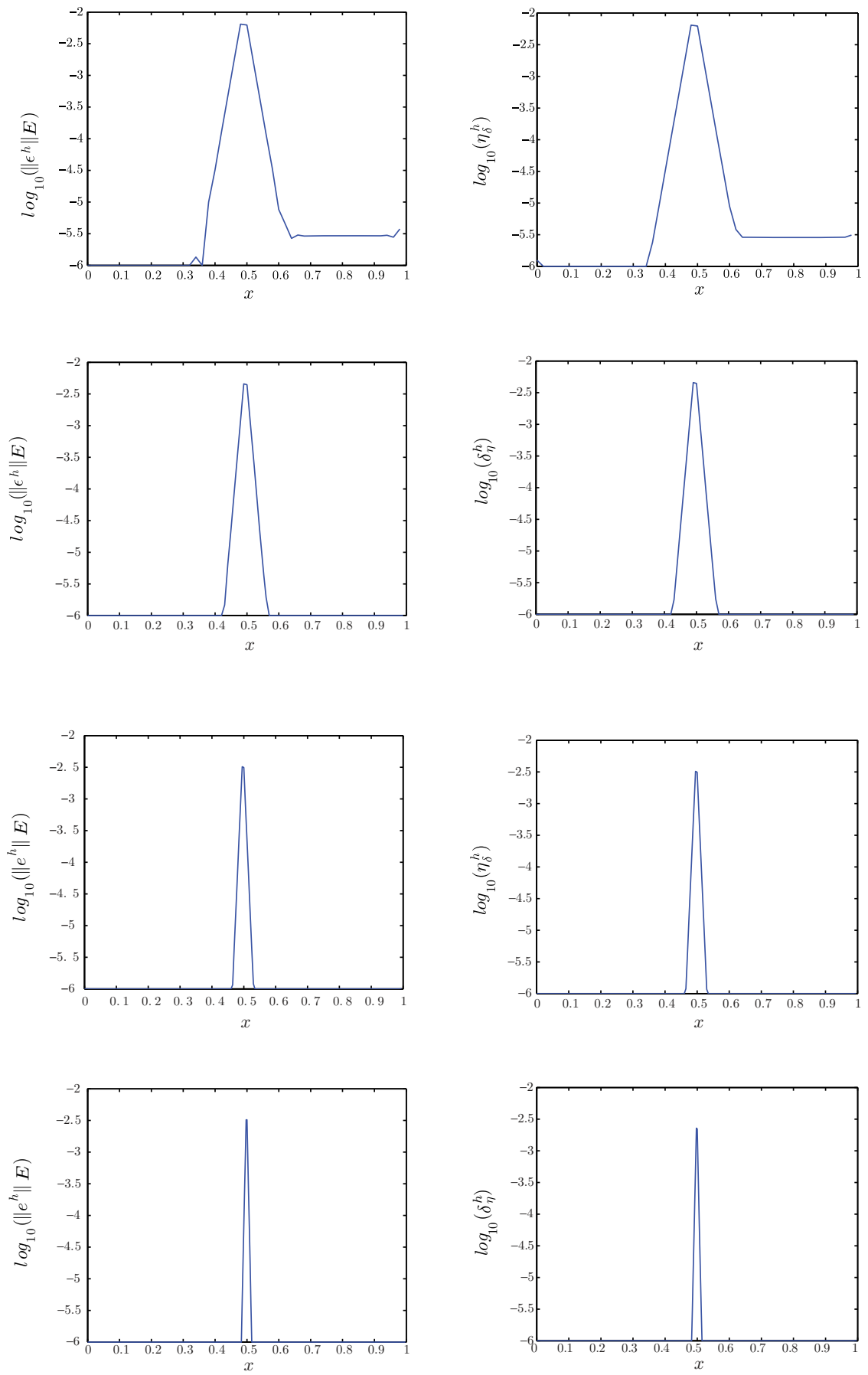

Figure 6. Error distributions of exact error $\left(\log _{10}\left(\left\|e^{h}\right\|_{E}\right)\right.$, left column) and estimator $\left(\log _{10}\left(\eta_{\delta}^{h}\right)\right.$, right column) for Example 5.2 with $\delta=2 ; N$ is taken to be 50,100, 200 and 400 from top to bottom, respectively. 

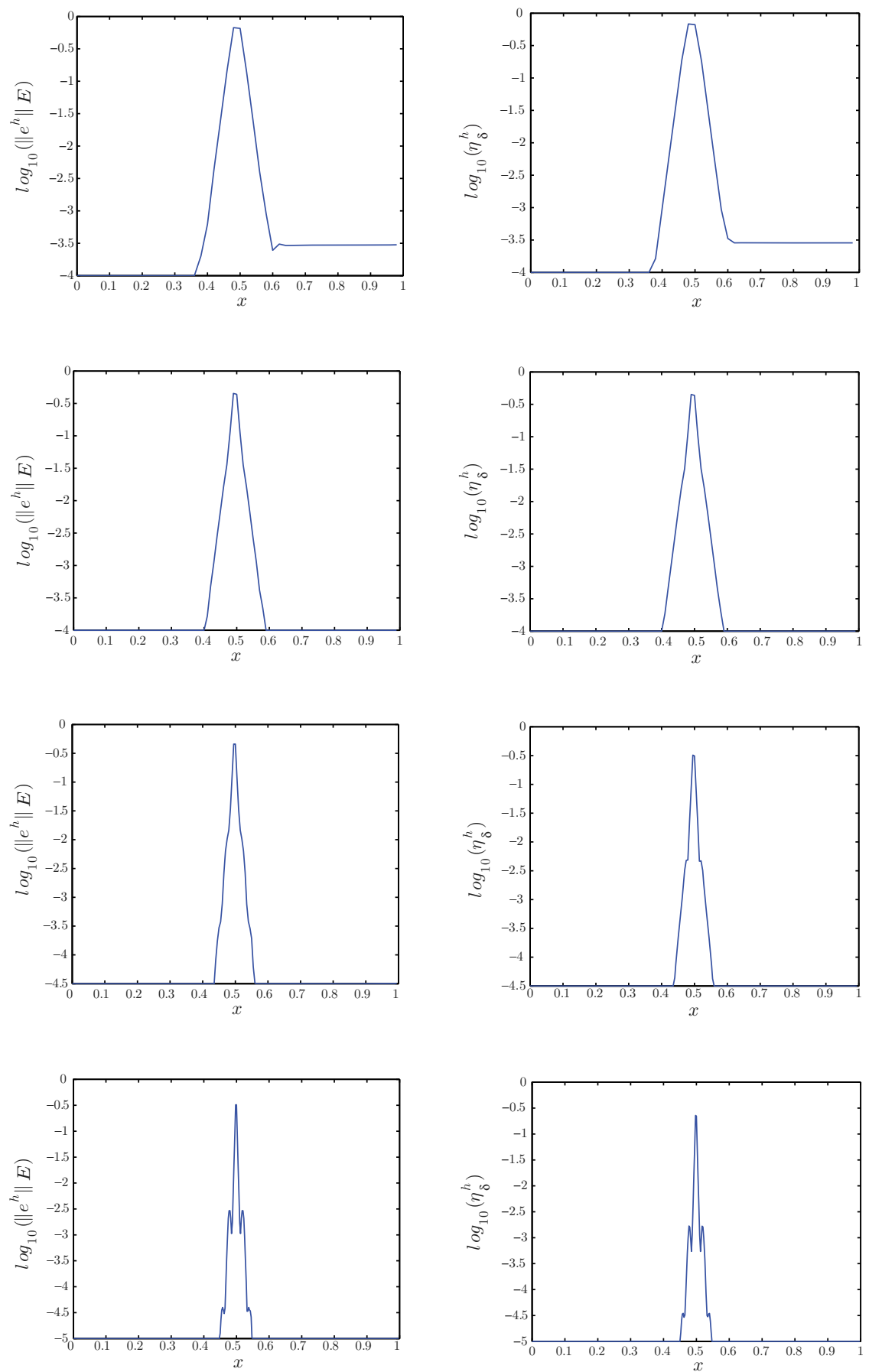

Figure 7. Error distributions of exact error $\left(\log _{10}\left(\left\|e^{h}\right\|_{E}\right)\right.$, left column) and estimator $\left(\log _{10}\left(\eta_{\delta}^{h}\right)\right.$, right column) for Example 5.2 with $\delta=0.02 ; N$ is taken to be 50,100, 200 and 400 from top to bottom, respectively. 
TABLE 3. Numerical results with different $\delta$ in Example 5.3

\begin{tabular}{|c|c|c|c|c|c|c|c|c|}
\hline \multicolumn{9}{|c|}{$\delta=2$} \\
\hline $\mathrm{N}$ & $\left\|e^{h}\right\|_{L^{2}}$ & $\mathrm{CR}$ & $\left\|e^{h}\right\|_{E}$ & $\mathrm{CR}$ & $\eta_{\delta}^{h}$ & $\mathrm{CR}$ & $\eta_{\delta}^{h} /\left\|e^{h}\right\|_{L^{2}}$ & $\eta_{\delta}^{h} /\left\|e_{h}\right\|_{E}$ \\
\hline 25 & $1.28 \mathrm{e}-02$ & - & $9.08 \mathrm{e}-03$ & - & $9.08 \mathrm{e}-03$ & - & 0.71 & 1.00 \\
\hline 50 & $3.90 \mathrm{e}-05$ & 8.36 & $1.50 \mathrm{e}-05$ & 9.24 & $1.50 \mathrm{e}-05$ & 9.24 & 0.38 & 1.00 \\
\hline 100 & $9.85 \mathrm{e}-06$ & 1.99 & $3.74 \mathrm{e}-06$ & 2.01 & $3.74 \mathrm{e}-06$ & 2.00 & 0.38 & 1.00 \\
\hline 200 & $2.49 \mathrm{e}-06$ & 1.99 & $9.36 \mathrm{e}-07$ & 2.00 & $9.35 \mathrm{e}-07$ & 2.00 & 0.38 & 1.00 \\
\hline 400 & $6.28 \mathrm{e}-07$ & 1.99 & $2.34 \mathrm{e}-07$ & 2.00 & $2.34 \mathrm{e}-07$ & 2.00 & 0.37 & 1.00 \\
\hline \multicolumn{9}{|c|}{$\delta=0.2$} \\
\hline $\mathrm{N}$ & $\left\|e^{h}\right\|_{L^{2}}$ & $\mathrm{CR}$ & $\left\|e^{h}\right\|_{E}$ & $\mathrm{CR}$ & $\eta_{\delta}^{h}$ & $\mathrm{CR}$ & $\eta_{\delta}^{h} /\left\|e^{h}\right\|_{L^{2}}$ & $\eta_{\delta}^{h} /\left\|e^{h}\right\|_{E}$ \\
\hline 25 & $1.28 \mathrm{e}-02$ & - & $9.07 \mathrm{e}-02$ & - & $9.08 \mathrm{e}-02$ & - & 7.07 & 1.00 \\
\hline 50 & $4.19 \mathrm{e}-05$ & 8.26 & $1.49 \mathrm{e}-04$ & 9.25 & $1.49 \mathrm{e}-04$ & 9.25 & 3.56 & 1.00 \\
\hline 100 & $1.06 \mathrm{e}-05$ & 1.98 & $3.73 \mathrm{e}-05$ & 2.00 & $3.73 \mathrm{e}-05$ & 2.00 & 3.51 & 1.00 \\
\hline 200 & $2.67 \mathrm{e}-06$ & 1.99 & $9.32 \mathrm{e}-06$ & 2.00 & $9.32 \mathrm{e}-06$ & 2.00 & 3.49 & 1.00 \\
\hline 400 & $6.72 \mathrm{e}-07$ & 1.99 & $2.33 \mathrm{e}-06$ & 2.00 & $2.33 \mathrm{e}-06$ & 2.00 & 3.47 & 1.00 \\
\hline \multicolumn{9}{|c|}{$\delta=0.02$} \\
\hline $\mathrm{N}$ & $\left\|e^{h}\right\|_{L^{2}}$ & $\mathrm{CR}$ & $\left\|e^{h}\right\|_{E}$ & $\mathrm{CR}$ & $\eta_{\delta}^{h}$ & $\mathrm{CR}$ & $\eta_{\delta}^{h} /\left\|e^{h}\right\|_{L^{2}}$ & $\eta_{\delta}^{h} /\left\|e^{h}\right\|_{E}$ \\
\hline 25 & $1.28 \mathrm{e}-02$ & - & $9.06 \mathrm{e}-01$ & - & $9.07 \mathrm{e}-01$ & - & 70.79 & 1.00 \\
\hline 50 & $4.29 \mathrm{e}-05$ & 8.22 & $1.49 \mathrm{e}-03$ & 9.25 & $1.49 \mathrm{e}-03$ & 9.25 & 34.75 & 1.00 \\
\hline 100 & $1.08 \mathrm{e}-05$ & 1.99 & $3.73 \mathrm{e}-04$ & 2.00 & $3.73 \mathrm{e}-04$ & 2.00 & 34.40 & 1.00 \\
\hline 200 & $2.72 \mathrm{e}-06$ & 1.99 & $9.32 \mathrm{e}-05$ & 2.00 & $9.32 \mathrm{e}-05$ & 2.00 & 34.25 & 1.00 \\
\hline 400 & $6.84 \mathrm{e}-07$ & 1.99 & $2.33 \mathrm{e}-05$ & 2.00 & $2.33 \mathrm{e}-05$ & 2.00 & 34.03 & 1.00 \\
\hline
\end{tabular}

Example 5.4. We use the same problem as in Example 5.1 but with a discontinuous exact solution whose discontinuity is inside one of the elements (for $N=25,50,100,200,400)$, defined as the following

$$
u(x)=\left\{\begin{array}{cc}
x, & x<0.503 \\
x^{2}, & \text { otherwise }
\end{array}\right.
$$

Therefore, $f(x)$ can be found as

$$
f(x)= \begin{cases}0, & x<0.503-\delta, \\ -\frac{1}{\delta^{3}}\left(\frac{(x+\delta)^{3}}{3}-\frac{(x+\delta)^{2}}{2}+\frac{1}{12}\right), & 0.503-\delta \leq x<0.503, \\ -\frac{1}{\delta^{3}}\left(\frac{(x-\delta)^{3}}{3}-\frac{(x-\delta)^{2}}{2}+\frac{1}{12}\right)-\frac{2}{3}, & 0.503 \leq x<0.503+\delta, \\ -\frac{2}{3}, & \text { otherwise. }\end{cases}
$$

We study the numerical solution with discontinuous piecewise linear basis functions and $\delta=2,0.2,0.02$. Computational results are listed in Table 4, one can observe that the convergence rates for the norms and estimator decrease to 0.5 , this is consistent with results in [20. Also our estimator approximates the exact error robustly. 
TABLE 4. Numerical results with different $\delta$ in Example 5.4

\begin{tabular}{|c|c|c|c|c|c|c|c|c|}
\hline \multicolumn{9}{|c|}{$\delta=2$} \\
\hline $\mathrm{N}$ & $\left\|e^{h}\right\|_{L^{2}}$ & $\mathrm{CR}$ & $\left\|e^{h}\right\|_{E}$ & $\mathrm{CR}$ & $\eta_{\delta}^{h}$ & $\mathrm{CR}$ & $\eta_{\delta}^{h} /\left\|e^{h}\right\|_{L^{2}}$ & $\eta_{\delta}^{h} /\left\|e^{h}\right\|_{E}$ \\
\hline 25 & $1.24 \mathrm{e}-02$ & - & $8.86 \mathrm{e}-03$ & - & $8.86 \mathrm{e}-03$ & - & 0.71 & 1.00 \\
\hline 50 & $9.77 \mathrm{e}-03$ & 0.35 & $6.90 \mathrm{e}-03$ & 0.36 & $6.90 \mathrm{e}-03$ & 0.36 & 0.71 & 1.00 \\
\hline 100 & $6.90 \mathrm{e}-03$ & 0.50 & $4.82 \mathrm{e}-03$ & 0.52 & $4.82 \mathrm{e}-03$ & 0.52 & 0.70 & 1.00 \\
\hline 200 & $5.08 \mathrm{e}-03$ & 0.44 & $3.41 \mathrm{e}-03$ & 0.50 & $3.41 \mathrm{e}-03$ & 0.50 & 0.67 & 1.00 \\
\hline 400 & $3.35 \mathrm{e}-03$ & 0.60 & $2.41 \mathrm{e}-03$ & 0.50 & $2.41 \mathrm{e}-03$ & 0.50 & 0.72 & 1.00 \\
\hline \multicolumn{9}{|c|}{$\delta=0.2$} \\
\hline $\mathrm{N}$ & $\left\|e^{h}\right\|_{L^{2}}$ & $\mathrm{CR}$ & $\left\|e^{h}\right\|_{E}$ & $\mathrm{CR}$ & $\eta_{\delta}^{h}$ & $\mathrm{CR}$ & $\eta_{\delta}^{h} /\left\|e^{h}\right\|_{L^{2}}$ & $\eta_{\delta}^{h} /\left\|e^{h}\right\|_{E}$ \\
\hline 25 & $1.25 \mathrm{e}-02$ & - & $8.86 \mathrm{e}-02$ & - & $8.86 \mathrm{e}-02$ & - & 7.07 & 1.00 \\
\hline 50 & $9.76 \mathrm{e}-03$ & 0.36 & $6.90 \mathrm{e}-02$ & 0.36 & $6.90 \mathrm{e}-02$ & 0.36 & 7.07 & 1.00 \\
\hline 100 & $6.82 \mathrm{e}-03$ & 0.52 & $4.83 \mathrm{e}-02$ & 0.52 & $4.83 \mathrm{e}-02$ & 0.52 & 7.07 & 1.00 \\
\hline 200 & $4.83 \mathrm{e}-03$ & 0.50 & $3.41 \mathrm{e}-02$ & 0.50 & $3.41 \mathrm{e}-02$ & 0.50 & 7.07 & 1.00 \\
\hline 400 & $3.42 \mathrm{e}-03$ & 0.50 & $2.41 \mathrm{e}-02$ & 0.50 & $2.41 \mathrm{e}-02$ & 0.50 & 7.07 & 1.00 \\
\hline \multicolumn{9}{|c|}{$\delta=0.02$} \\
\hline $\mathrm{N}$ & $\left\|e^{h}\right\|_{L^{2}}$ & $\mathrm{CR}$ & $\left\|e^{h}\right\|_{E}$ & $\mathrm{CR}$ & $\eta_{\delta}^{h}$ & $\mathrm{CR}$ & $\eta_{\delta}^{h} /\left\|e^{h}\right\|_{L^{2}}$ & $\eta_{\delta}^{h} /\left\|e^{h}\right\|_{E}$ \\
\hline 25 & $1.24 \mathrm{e}-02$ & - & $8.86 \mathrm{e}-01$ & - & $8.88 \mathrm{e}-01$ & - & 71.61 & 1.00 \\
\hline 50 & $9.62 \mathrm{e}-03$ & 0.37 & $6.90 \mathrm{e}-01$ & 0.36 & $6.90 \mathrm{e}-01$ & 0.36 & 71.78 & 1.00 \\
\hline 100 & $6.87 \mathrm{e}-03$ & 0.48 & $4.82 \mathrm{e}-01$ & 0.52 & $4.83 \mathrm{e}-01$ & 0.52 & 70.24 & 1.00 \\
\hline 200 & $4.81 \mathrm{e}-03$ & 0.52 & $3.41 \mathrm{e}-01$ & 0.50 & $3.43 \mathrm{e}-01$ & 0.49 & 71.30 & 1.01 \\
\hline 400 & $3.49 \mathrm{e}-03$ & 0.50 & $2.41 \mathrm{e}-01$ & 0.50 & $2.41 \mathrm{e}-01$ & 0.51 & 70.83 & 1.00 \\
\hline
\end{tabular}

Example 5.5. In the last example, we take a singular kernel function by choosing $\boldsymbol{\alpha}=\frac{x^{\prime}-x}{\left|x^{\prime}-x\right|}$ and $\beta=-\frac{1}{\delta^{2} \sqrt{\delta\left|x^{\prime}-x\right|}}$, consider the following problem.

$$
\left\{\begin{aligned}
-\frac{1}{\delta^{2} \sqrt{\delta}} \int_{x-\delta}^{x+\delta} \frac{u\left(x^{\prime}\right)-u(x)}{\sqrt{\left|x^{\prime}-x\right|}} d x^{\prime} & =f(x), & & x \in[0,1], \\
u(x) & =g(x), & & x \in[-\delta, 0] \cup[1,1+\delta] .
\end{aligned}\right.
$$

We set the exact solution,

$$
u(x)=x^{2},
$$

and therefore

$$
f(x)=-\frac{4}{5} .
$$

The boundary term $g(x)$ is decided from equation (5.6). We solve the problem with $\delta=2,0.2$, respectively.

As in previous examples, we use the continuous Galerkin method with piecewise linear basis functions. Again, for fixed $\delta$, we divide the interval $[0,1]$ into $N=$ 25, 50, 100, 200, 400 subintervals, respectively. Numerical results are shown in Table 5. We again can observe that the error estimator has the same convergence rate with $\left\|e^{h}\right\|_{E}$, and the ratio between the two is very stable, where the efficiency can be observed. For the kernel function used in this particular example, we also have

$$
\sqrt{M(\delta)} \sim \sqrt{\frac{1}{\delta^{2} \sqrt{\delta}} \int_{-\delta}^{\delta} \frac{1}{\sqrt{|z|}} d z} \sim \frac{1}{\delta}
$$


TABle 5. Numerical results with different $\delta$ in Example 5.5

\begin{tabular}{|c||c|c||c|c||c|c||c|c|}
\hline \multicolumn{10}{|c|}{$\delta=2$} \\
\hline $\mathrm{N}$ & $\left\|e^{h}\right\|_{L^{2}}$ & $\mathrm{CR}$ & $\left\|e^{h}\right\|_{E}$ & $\mathrm{CR}$ & $\eta_{\delta}^{h}$ & $\mathrm{CR}$ & $\eta_{\delta}^{h} /\left\|e^{h}\right\|_{L^{2}}$ & $\eta_{\delta}^{h} /\left\|e_{h}\right\|_{E}$ \\
\hline 25 & $2.93 \mathrm{e}-04$ & - & $1.17 \mathrm{e}-04$ & - & $1.16 \mathrm{e}-04$ & - & 0.40 & 0.99 \\
\hline 50 & $7.31 \mathrm{e}-05$ & 2.00 & $2.95 \mathrm{e}-05$ & 1.99 & $2.96 \mathrm{e}-05$ & 1.97 & 0.41 & 1.00 \\
\hline 100 & $1.83 \mathrm{e}-05$ & 2.00 & $7.40 \mathrm{e}-06$ & 2.00 & $7.59 \mathrm{e}-06$ & 1.96 & 0.42 & 1.03 \\
\hline 200 & $4.57 \mathrm{e}-06$ & 2.00 & $1.85 \mathrm{e}-06$ & 2.00 & $1.97 \mathrm{e}-06$ & 1.95 & 0.43 & 1.06 \\
\hline 400 & $1.14 \mathrm{e}-06$ & 2.00 & $4.63 \mathrm{e}-07$ & 2.00 & $4.91 \mathrm{e}-07$ & 2.00 & 0.43 & 1.06 \\
\hline \multicolumn{10}{|c|}{$\delta=0.2$} \\
\hline $\mathrm{N}$ & $\left\|e^{h}\right\|_{L^{2}}$ & $\mathrm{CR}$ & $\left\|e^{h}\right\|_{E}$ & $\mathrm{CR}$ & $\eta_{\delta}^{h}$ & $\mathrm{CR}$ & $\eta_{\delta}^{h} /\left\|e^{h}\right\|_{L^{2}}$ & $\eta_{\delta}^{h} /\left\|e^{h}\right\|_{E}$ \\
\hline 25 & $3.13 \mathrm{e}-04$ & - & $1.13 \mathrm{e}-03$ & - & $1.18 \mathrm{e}-03$ & - & 3.75 & 1.04 \\
\hline 50 & $7.68 \mathrm{e}-05$ & 2.03 & $2.88 \mathrm{e}-04$ & 1.98 & $2.97 \mathrm{e}-04$ & 1.99 & 3.86 & 1.03 \\
\hline 100 & $1.89 \mathrm{e}-05$ & 2.02 & $7.28 \mathrm{e}-05$ & 1.98 & $7.51 \mathrm{e}-05$ & 1.98 & 3.97 & 1.03 \\
\hline 200 & $4.69 \mathrm{e}-06$ & 2.02 & $1.83 \mathrm{e}-05$ & 1.99 & $1.89 \mathrm{e}-05$ & 1.99 & 4.04 & 1.03 \\
\hline 400 & $1.16 \mathrm{e}-06$ & 2.01 & $4.60 \mathrm{e}-06$ & 1.99 & $4.74 \mathrm{e}-06$ & 2.00 & 4.08 & 1.03 \\
\hline
\end{tabular}

which again explains the growth of $\eta_{\delta}^{h} /\left\|e^{h}\right\|_{L^{2}}$.

\subsection{Two-dimensional numerical experiments.}

Example 5.6. For given horizon parameter $\delta$, denote $\hat{\Omega}_{s}=[0,1] \times[0,1], \hat{\Omega}=$ $[-\delta, 1+\delta] \times[-\delta, 1+\delta]$. We consider the following $2 \mathrm{D}$ nonlocal problem, with $\boldsymbol{\alpha}=\frac{\mathbf{x}^{\prime}-\mathbf{x}}{\left|\mathbf{x}^{\prime}-\mathbf{x}\right|}$ and $\beta=-\frac{1}{\delta^{4}}$,

$$
\left\{\begin{aligned}
-\frac{1}{\delta^{4}} \int_{B_{\delta}(\mathbf{x})}\left(u\left(\mathbf{x}^{\prime}\right)-u(\mathbf{x})\right) d x^{\prime} & =f(\mathbf{x}), & & \mathbf{x} \in \hat{\Omega}_{s}, \\
u(\mathbf{x}) & =g(\mathbf{x}), & & \mathbf{x} \in \hat{\Omega} / \hat{\Omega}_{s} .
\end{aligned}\right.
$$

We choose the discontinuous exact solution to be

$$
u(\mathbf{x})=\left\{\begin{aligned}
x+y, & x<0.8333 \\
x^{2}+y^{2}, & \text { otherwise }
\end{aligned}\right.
$$

where $\mathbf{x}=(x, y)$. Then $f(\mathbf{x})$ and $g(\mathbf{x})$ can be decided accordingly, and we can choose

$$
M(\delta)=\int_{B_{\delta}(0)} \frac{1}{\delta^{4}} d \mathbf{x}=\frac{\pi}{\delta^{2}} .
$$

In this experiment, we use the discontinuous Galerkin method with piecewise constant basis functions, and take $\delta=0.1,0.2$, respectively. For each fixed $\delta$, we find numerical solutions over $N \times N$ uniform square meshes, where $N=10,20,40$ and 80 , respectively. Notice that under this setting, the discontinuities fall inside grid elements, which is general since one does not need to know the positions of discontinuities in advance.

Figure 8 shows distributions of $\log _{10}\left(\left\|e^{h}\right\|_{E}\right)$ and $\log _{10}\left(\eta^{h}\right)$ for all the grids over $[0,1] \times[0,1]$, with $\delta=0.1$ and $N=20$. It can be observed that our error estimator matches the exact error very well, the relatively large errors around discontinuities are captured. Convergence results are listed in Table [6] one can observe that the convergence rate is 0.5 and the estimator approximates the exact energy norm error robustly. 

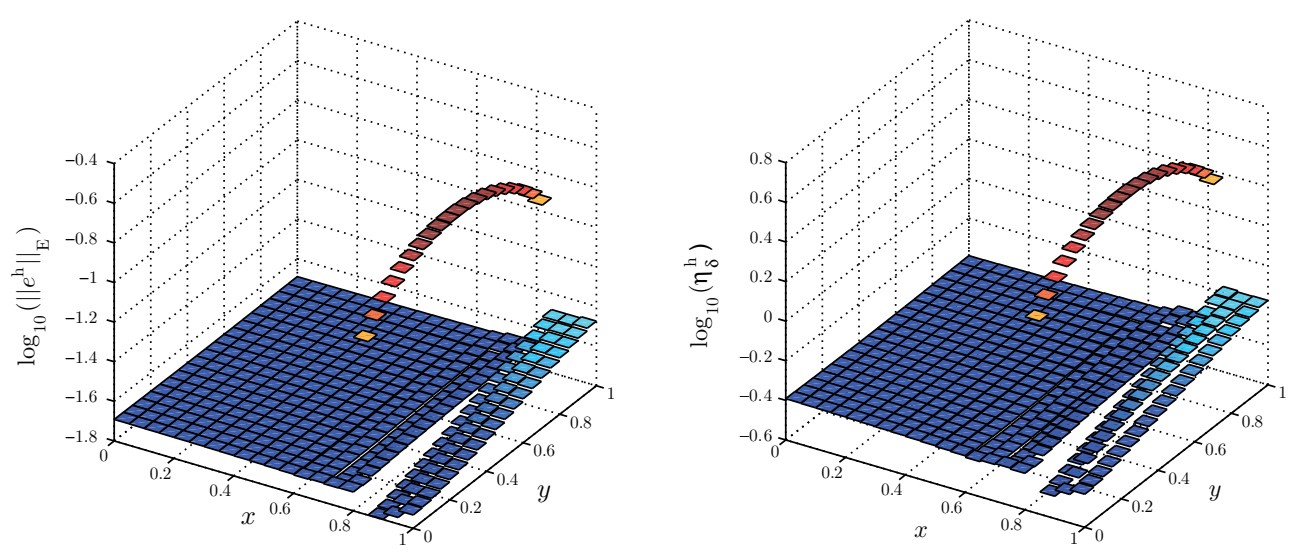

Figure 8. Error distributions of exact error $\left(\log _{10}\left(\left\|e^{h}\right\|_{E}\right)\right.$, left $)$ and estimator $\left(\log _{10}\left(\eta_{\delta}^{h}\right)\right.$, right) for Example 5.2 with $\delta=0.1$, $20 \times 20 \operatorname{mesh}(N=20)$.

TABLE 6. Numerical results with different $\delta$ in Example 5.6

\begin{tabular}{|c||c|c||c|c||c|c||c|c|}
\hline \multicolumn{10}{|c|}{$\delta=0.2$} \\
\hline $\mathrm{N}$ & $\left\|e^{h}\right\|_{L^{2}}$ & $\mathrm{CR}$ & $\left\|e^{h}\right\|_{E}$ & $\mathrm{CR}$ & $\eta_{\delta}^{h}$ & $\mathrm{CR}$ & $\eta_{\delta}^{h} /\left\|e^{h}\right\|_{L^{2}}$ & $\eta_{\delta}^{h} /\left\|e_{h}\right\|_{E}$ \\
\hline 10 & $7.0078 \mathrm{e}-02$ & - & $6.9594 \mathrm{e}-01$ & - & $7.8443 \mathrm{e}-01$ & - & 11.19 & 1.13 \\
\hline 20 & $4.7783 \mathrm{e}-02$ & 0.55 & $4.7410 \mathrm{e}-01$ & 0.55 & $5.3456 \mathrm{e}-01$ & 0.55 & 11.19 & 1.13 \\
\hline 40 & $3.2846 \mathrm{e}-02$ & 0.54 & $3.2822 \mathrm{e}-01$ & 0.53 & $3.7036 \mathrm{e}-01$ & 0.53 & 11.28 & 1.13 \\
\hline 80 & $2.2966 \mathrm{e}-02$ & 0.52 & $2.2950 \mathrm{e}-01$ & 0.52 & $2.5896 \mathrm{e}-01$ & 0.52 & 11.28 & 1.13 \\
\hline \multicolumn{10}{|c|}{$\delta=0.1$} \\
\hline $\mathrm{N}$ & $\left\|e^{h}\right\|_{L^{2}}$ & $\mathrm{CR}$ & $\left\|e^{h}\right\|_{E}$ & $\mathrm{CR}$ & $\eta_{\delta}^{h}$ & $\mathrm{CR}$ & $\eta_{\delta}^{h} /\left\|e^{h}\right\|_{L^{2}}$ & $\eta_{\delta}^{h} /\left\|e^{h}\right\|_{E}$ \\
\hline 10 & $7.5791 \mathrm{e}-02$ & - & $1.3561 \mathrm{e}+00$ & - & $1.5005 \mathrm{e}+00$ & - & 19.80 & 1.11 \\
\hline 20 & $5.2745 \mathrm{e}-02$ & 0.52 & $9.4233 \mathrm{e}-01$ & 0.53 & $1.0575 \mathrm{e}+00$ & 0.50 & 20.05 & 1.12 \\
\hline 40 & $3.3323 \mathrm{e}-02$ & 0.66 & $6.5578 \mathrm{e}-01$ & 0.52 & $7.3942 \mathrm{e}-01$ & 0.52 & 22.19 & 1.13 \\
\hline 80 & $2.3269 \mathrm{e}-02$ & 0.52 & $4.5888 \mathrm{e}-01$ & 0.52 & $5.1766 \mathrm{e}-01$ & 0.51 & 22.25 & 1.13 \\
\hline
\end{tabular}

\section{Conclusions}

In this work, a rigorous mathematical framework is established for the a posteriori error analysis of the finite element solutions for nonlocal diffusion and nonlocal peridynamic equations. The estimators are carefully derived and the reliability and efficiency are proven. The numerical experiments are consistent with our theoretical conclusion. Meanwhile, the weak convergence of the nonlocal estimator to its local analogue is demonstrated for nonlocal diffusion equation and peridynamic model problem, drawing interesting connections to the limit case for which many of studies can be found in the literature. The nonlocal nature of the problem also offers some sharp contrasts with the local counterpart as explored in our analysis. While the analysis is done for problems with an operator-induced norm being equivalent to $L^{2}$ norm, due to our choice of kernel function, it is possible to generalize such a framework of a posteriori error analysis for problems involving more general kernel functions. This will be pursued in our future works, along with the 
development of the adaptive convergence analysis and practical implementation for nonlocal models.

\section{ACKNOWLEDGMENTS}

The authors would like to thank the referees for their constructive comments that have helped us to improve the manuscript.

\section{REFERENCES}

1. M. Ainsworth and J. Oden, A posteriori error estimation in finite element analysis, Wiley, New York, 2002. MR.1885308 (2003b:65001)

2. B. Aksoylu and M. Parks, Variational theory and domain decomposition for nonlocal problems, Applied Mathematics and Computation 217 (2011), 6498-6515. MR2773238 (2012d:65306)

3. B. Aksoylu and T. Mengesha, Results on nonlocal boundary value problems, Numerical Functional Analysis and Optimization 31 (2010), 1301-1317. MR2738853 (2011m:35395)

4. B. Alali and R. Lipton, Multiscale Dynamics of Heterogeneous Media in the Peridynamic formulation, J. Elasticity (2010), DOI: 10. 1007/s10659-010-9291-4. MR2870298

5. F. Andreu, J. M. Mazón, J. D. Rossi, and J. Toledo. A nonlocal $p$-Laplacian evolution equation with nonhomogeneous Dirichlet boundary conditions. SIAM J. Math. Anal. 40 (2009), 18151851. MR2471902 (2011a:35542)

6. E. Askari, F. Bobaru, R. B. Lehoucq, M. L. Parks, S. A. Silling, and O. Weckner, Peridynamics for multiscale materials modeling, J. Phys. : Conf. Ser. 125 (2008), DOI:10. 1088/17426596/125/1/012078.

7. G. Aubert and P. Kornprobst. Can the nonlocal characterization of Sobolev spaces by bourgain et al. be useful for solving variational problems?, SIAM J. Numer. Anal. 47 (2009), 844-860. MR2485435 (2010c:49005)

8. I. Babuska and A. Miller, A feedback finite element method with a posteriori error estimates, Comput. Methods Appl. Mech. Engrg. 61 (1987), 1-40. MR880421 (88d:73036)

9. I. Babuska and W. C. Rheinboldt, A posteriori error estimates for the finite element method, Internat. J. Numer. Methods Engrg. 12 (1978), 1597-1615.

10. I. Babuska and W. C. Rheinboldt, A posteriori error analysis of finite element solutions for one-dimensional problems, SIAM J. Numer. Anal. 18 (1981), 565-589. MR615532|(82j:65082)

11. I. Babuska and W. C. Rheinboldt, Error estimates for adaptive finite element computations, SIAM J. Numer. Anal. 15 (1987), 736-754. MR0483395 (58:3400)

12. S. Bartels, C. Carstensen, and G. Dolzmann, Inhomogeneous dirichlet conditions in a priori and a posteriori finite element error analysis, Numer. Math. 99 (2004), 1-24. MR.2101782 (2005i:65166)

13. F. Bobaru, L. A. Alves, S. A. Silling, and A. Askari, Dynamic crack branching and adaptive refinement in peridynamics, 8th World Congress on Computational Mechanics, 5th European Congress on Computational Methods in Applied Sciences and Engineering, June 30-July 5, 2008, Venice, Italy.

14. F. Bobaru, M. Yang, L. F. Alves, S. A. Silling, E. Askari, and J. Xu, Convergence, adaptive refinement, and scaling in 1d peridynamics, Intern. J. Numer. Meth. Engrg. 77 (2009), 852877 .

15. J. Bourgain, H. Brezis, and P. Mironescu, Another look at Sobolev spaces, in: J. L. Menaldi, E. Rofman, A. Sulem (Eds. ), Optimal Control and Partial Differential Equations. A Volume in Honour of A. Bensoussan's 60th Birthday, IOS Press (2001), 439-455.

16. N. Burch and R. Lehoucq, Classical, nonlocal, and fractional diffusion equations on bounded domains, Sandia Technical Report, SAND 2010-1762J, Sandia National Laboratories, 2010.

17. C. Carstensen, Efficiency of a posteriori error estimates for first-kind integral equations on quasi-uniform meshes, Math. Comp. 65 (1996), 69-84. MR.1320892 (96j:65145)

18. C. Carstensen, All first-order averaging techniques for a posteriori finite element error control on unstructured grids are efficient and reliable, Math. Comp. 73 (2003), 1153-1165. MR2047082 (2005e:65173)

19. C. Carstensen and B. P. Rynne, A posteriori error control for finite element approximations of the integral equation for thin wire antennas, Z. Angew. Math. Mech. 82 (2002), 284-288. MR1900489 (2003c:65145) 
20. X. Chen and M. Gunzburger, Continuous and discontinuous finite element methods for a peridynamics model of mechanics, Comput. Methods Appl. Mech. Engrg. 200 (2010), 12371250. MR2796157 (2011m:65167)

21. A. Demlow, Local a posteriori estimates for pointwise gradient errors in finite element methods for elliptic problems, Math. Comp. 76 (2007), 19-42. MR.2261010 (2008c:65319)

22. Q. Du, M. Gunzburger, R. B. Lehoucq, and K. Zhou, A nonlocal vector calculus, nonlocal volume-constrained problems, and the nonlocal balance laws, Math. Mod. Meth. Appl. Sci. 23 (2013), pp. 493-540.

23. Q. Du, M. Gunzburger, R. B. Lehoucq, and K. Zhou, Analysis and approximation of nonlocal diffusion problems with volume constraints, SIAM Review 56 (2012), pp. 676-696.

24. Q. Du, M. Gunzburger, R. B. Lehoucq, and K. Zhou, Analysis of the volume-constrained peridynamic Navier equation of linear elasticity, Journal of Elasticity, 2013 (online), doi:10.1007/s10659-012-9418-x.

25. Q. Du and K. Zhou, Mathematical analysis for the peridynamics nonlocal continuum theory, ESAIM: M2AN 45 (2011), 217-234. MR2804637

26. E. Emmrich and O. Weckner, Analysis and numerical approximation of an integrodifferential equation modeling non-local effects in linear elasticity, Math. Mech. Solids 12 (2005), 363-384. MR2349159(2008i:74052)

27. E. Emmrich and O. Weckner, The peridynamic equation of motion in nonlocal elasticity theory, III European Conference on Computational Mechanics - Solids, Structures and Coupled Problems in Engineering, 2006.

28. E. Emmrich and O. Weckner, On the well-posedness of the linear peridynamic model and its convergence towards the navier equation of linear elasticity, Commun. Math. Sci. 5 (2007), 851-864. MR2375050(2008m:74061)

29. M. Gunzburger and R. B. Lehoucq, A nonlocal vector calculus with application to nonlocal boundary value problems, Multiscale Model. Simul. 8 (2010), 1581-1598. MR2728700 (2012c:35094)

30. L. Ju, M. Gunzburger, and W. Zhao, Adaptive finite element methods for elliptic pdes based on conforming centroidal Voronoi-Delaunay triangulations, SIAM J. Sci. Comput. 28 (2003), 2023-2053. MR.2272250 (2007k:65182)

31. R. B. Lehoucq and S. A Silling, Force flux and the peridynamic stress tensor, J. Mech. Phys. Solids 56 (2008), 1566-1577. MR2404022 (2009c:74003)

32. R. W. Macek and S. A. Silling, Peridynamics via finite element analysis, Finite Elements in Analysis and Design 43 (2007), 1169-1178. MR2393568

33. R. H. Nochetto, T. Petersdorff, and C. -S. Zhang, A posteriori error analysis for a class of integral equations and variational inequalities, Numer. Math. 116 (2010), 519-552. MR2684296 (2011g:65103)

34. S. A. Silling, Reformulation of elasticity theory for discontinuities and long-range forces, J. Mech. Phys. Solids 48 (2000), 175-209. MR.1727557 (2000i:74008)

35. S. A. Silling, A coarsening method for linear peridynamics, International Journal for Multiscale Computational Engineering 9 (2011), pp. 609-622.

36. S. A. Silling and E. Askari, A meshfree method based on the peridynamic model of solid mechanics, Computers \& Structures 83 (2005), Advances in Meshfree Methods, 1526-1535.

37. S. A. Silling and R. B. Lehoucq, Convergence of peridynamics to classical elasticity theory, J. Elasticity 93 (2008), 13-37. MR2430855 (2009i:74003)

38. R. Verfürth, a review of a posteriori error estimation and adaptive mesh-refinement techniques, Wiley-Teubner, Chichester, UK, 1996.

39. K. Yu, X. J. Xin, and K. B. Lease, A new method of adaptive integration with error control for bond-based peridynamics, Proceedings of the World Congress on Engineering and Computer Science 2010, Vol II, San Francisco, USA.

40. K. Zhou and Q. Du, Mathematical and numerical analysis of linear peridynamic models with nonlocal boundary conditions, SIAM J. Numer. Anal. 48 (2010), 1759-1780. MR2733097 (2011k:82057) 
Department of Mathematics, Pennsylvania State University, University Park, PennSYLVANIA 16802

E-mail address: qdu@math.psu.edu

Department of Mathematics, University of South Carolina, Columbia, South CarOLINA 29208

E-mail address: ju@math.sc.edu

Department of Mathematics, Pennsylvania State University, University Park, PennSYLVANIA 16802

E-mail address: tian@math.psu.edu

Department of Mathematics, Pennsylvania State University, University Park, PennSYLVANIA 16802

E-mail address: zhou@math.psu.edu 